\title{
Chaotic Motion in the Breathing Circle Billiard
}

\author{
Claudio Bonanno® and Stefano Marò®
}

\begin{abstract}
We consider the free motion of a point particle inside a circular billiard with periodically moving boundary, with the assumption that the collisions of the particle with the boundary are elastic so that the energy of the particle is not preserved. It is known that if the motion of the boundary is regular enough then the energy is bounded due to the existence of invariant curves in the phase space. We show that it is nevertheless possible that the motion of the particle is chaotic, also under regularity assumptions for the moving boundary. More precisely, we show that there exists a class of functions describing the motion of the boundary for which the billiard map has positive topological entropy. The proof relies on variational techniques based on the Aubry-Mather theory.
\end{abstract}

Mathematics Subject Classification. 37C83, 37B40, 37E40.

\section{Introduction}

A mathematical billiard with moving boundary is a region of the plane instantaneously bounded by a closed curve changing with time. The billiard problem then consists of the free motion of a point particle inside this region colliding elastically with the moving boundary.

The study of time-dependent billiards can be motivated physically by the study of confined Lorentz gas or by some models in nuclear physics (see, for instance, $[5,6,13,31])$. In the physical contexts, a relevant question is whether the elastic bounces can make the energy of the particle grow infinitely. The question was raised by Fermi [10] trying to explain the high velocity gained by photons. A mathematical formulation of the problem was proposed by Ulam and is now called the Fermi-Ulam model. It describes the free motion of a particle between two parallel walls moving periodically. "Fermi acceleration" 
then occurs if the elastic bounces with the moving walls make the energy of the particle tend to infinity. It is known that it depends on the regularity of the motion of the walls. Actually, it was proved in [17] that if the motion of the walls is at least $C^{6}$ then KAM theory applies and the energy remains bounded. On the other hand, in [33] it is shown how to construct a motion of the walls that is only continuous and allows the energy to grow to infinity (see also [21] for a similar result in a related impact model).

Time-dependent billiards can be seen as natural generalisations of the Fermi-Ulam model, and the question on the existence of Fermi acceleration naturally extends to this context. In this case, the answer also depends on the geometry of the boundary.

If the boundary is a moving ellipse, then it has been proved in [9] that it is possible to construct orbits that gain energy. Moreover, the existence of unbounded motions is a symptom of complex dynamics. In fact, in [9] it is also proved that the phenomenon of splitting of the separatrices occurs and a scattering map can be defined. We refer to [12] for more insight on the topic of Fermi acceleration in general time-dependent billiards.

Instead, if the boundary is a circle with radius varying smoothly with time, then KAM theory applies and the energy remains bounded [29]. Since the motion along a diameter is described by the classical Fermi-Ulam model, the counterexample constructed in [33] shows that the regularity of the motion of the boundary is a fundamental assumption in [29].

In this paper, we deal with the case of a region with circular boundary of radius $R(t)$ periodic in time. The region is called the breathing circle billiard. We show that chaotic phenomena can occur also if the motion of the boundary is regular. More precisely, we find a class of function $\widetilde{\mathcal{R}}$ such that if $R \in \tilde{\mathcal{R}}$ then the associated billiard map has positive topological entropy. The class $\widetilde{\mathcal{R}}$ has a somehow technical definition, but it can be shown that a representative is

$$
R(t)=M+\delta \sin (2 \pi t)
$$

with $M$ sufficiently large with respect to $\delta$.

The dynamics of a time-dependent billiard whose boundary remains a convex curve can be described by a 4-dimensional exact symplectic map [15]. However, for the breathing circle billiard, the angular momentum is a first integral so that the dynamics can be reduced to a two-dimensional map of the cylinder that turns out to be exact symplectic and twist at large energies. We show that to the reduced map we can apply the variational framework of Aubry-Mather theory, which implies the existence of interesting invariant sets. In particular, a key role is played by invariant curves with irrational rotation numbers. More precisely, it is known that the lack of invariant curves for a given irrational rotation number implies the existence of chaotic motion, and in particular we apply a result by Forni [11] to obtain the existence of many invariant measures with positive metric entropy (and support of zero Lebesgue measure), from which positiveness of topological entropy follows. See $[1,2]$ for related results. 
In the last decades, many results have been proved in the direction of "breaking" invariant curves, also in higher dimensions, giving rise to the so-called converse KAM theory $[14,18,19]$. In this paper, we show the nonexistence of some invariant curve using a criterion based on the variational approach of Aubry-Mather theory, in the spirit of what is done in [24]. More precisely, it is known that orbits of exact symplectic twist maps correspond to stationary points of an action functional, and the ones on invariant curves are minimal. As a consequence, the second variation of the action must be positive on orbits lying on invariant curves. The main idea to prove our main result is then to show that if $R \in \widetilde{\mathcal{R}}$ the second variation of the action is negative in a zone of the phase space, preventing the existence of invariant curves for some irrational rotation numbers. From a technical point of view, in order to compute the second variation of the action, one needs the generating function of the associated diffeomorphism. A considerable part of the paper is dedicated to get an explicit formulation of the generating function of the billiard map for large energies. To this aim, we follow the idea, used in [16] for the (non-periodic) Fermi-Ulam model, that the generating function is given by the Lagrangian action of a solution of the Dirichlet problem between two consecutive impacts. We conclude noting that a consequence of our approach is the existence of Aubry-Mather sets with different rotation numbers, giving rise to periodic and quasi-periodic motions of the breathing circle billiard. Similar results have been obtained for other systems with impacts such as bouncing balls [20].

The paper is organised as follows: In Sect. 2, we state the problem and the main results of the paper. In Sect.3, we study the Dirichlet problem between two consecutive impacts, and its results are used in Sect. 4 to compute the generating function of the billiard map. In Sect. 5 , we describe the periodic and quasi-periodic motions. Section 6 is dedicated to the proof of the main theorem on chaotic motion. Appendix A contains the proof that the class of functions $\widetilde{\mathcal{R}}$ is not empty. Finally, the main results of Aubry-Mather theory used in the paper are collected in Appendix B.

\section{Statement of the Problem and Main Results}

Let $R(t)$ be a strictly positive function, and let the breathing circle $\mathcal{D}_{t}$ be the bounded region of the plane with moving boundary $\partial \mathcal{D}_{t}=\left\{x \in \mathbb{R}^{2}:|x|^{2}=\right.$ $\left.R^{2}(t)\right\}$. Let us consider a particle of unitary mass moving freely inside $\mathcal{D}_{t}$ and satisfying the elastic impact law at every bounce on the boundary. Assume that $\partial \mathcal{D}_{t}$ is positively oriented and denote by $\hat{\tau}, \hat{\eta}$ the unitary tangent and outward normal vectors at points of $\partial \mathcal{D}_{t}$. By a bouncing solution, we mean a continuous and piecewise- $C^{2}$ function

$$
x: \mathbb{R} \rightarrow \mathbb{R}^{2}
$$

with a sequence of impact times $\left(t_{n}\right)_{n \in \mathbb{Z}}$ satisfying

1. $\ddot{x}(t)=0$ for $t \in\left(t_{n}, t_{n+1}\right)$ for every $n \in \mathbb{Z}$,

2. $|x(t)|<R(t)$ for $t \in\left(t_{n}, t_{n+1}\right)$ for every $n \in \mathbb{Z}$, 
3. $\left|x\left(t_{n}\right)\right|=R\left(t_{n}\right)$ for every $n \in \mathbb{Z}$,

4. $\dot{x}\left(t_{n}^{+}\right) \cdot \hat{\tau}_{n}=\dot{x}\left(t_{n}^{-}\right) \cdot \hat{\tau}_{n}$ and $\dot{x}\left(t_{n}^{+}\right) \cdot \hat{\eta}_{n}=-\dot{x}\left(t_{n}^{-}\right) \cdot \hat{\eta}_{n}+2 \dot{R}\left(t_{n}\right)$, where $\hat{\tau}_{n}, \hat{\eta}_{n}$ are the unitary tangent and outward normal vectors at $x\left(t_{n}\right)$, and $\dot{x}\left(t_{n}^{-}\right)$ and $\dot{x}\left(t_{n}^{+}\right)$denote the velocity vector just before and after the bounce at time $t_{n}$, respectively.

Condition 4 describes the elastic bouncing condition: the tangent component of the velocity is preserved and the impulse is given in the normal direction. Note that if $\dot{R} \equiv 0$ we get the usual mirror law.

To describe the possible bouncing solutions, we first note that the angular momentum is preserved, more precisely,

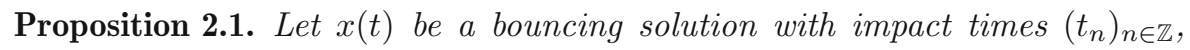
and then, the angular momentum $C(t)=x(t) \wedge \dot{x}(t)$ is constant for every $t$.

Proof. It is clear that the angular momentum is constant for $t \in\left(t_{n}, t_{n+1}\right)$ for every $n \in \mathbb{Z}$. Moreover at the bouncing time it holds

$$
\begin{aligned}
C\left(t_{n}^{-}\right) & =x\left(t_{n}\right) \wedge \dot{x}\left(t_{n}^{-}\right)=R\left(t_{n}\right) \hat{\eta}_{n} \wedge \dot{x}\left(t_{n}^{-}\right)=R\left(t_{n}\right) \hat{\eta}_{n} \wedge\left(\dot{x}\left(t_{n}^{-}\right) \cdot \hat{\tau}_{n}\right) \hat{\tau}_{n} \\
& =R\left(t_{n}\right) \hat{\eta}_{n} \wedge\left(\dot{x}\left(t_{n}^{+}\right) \cdot \hat{\tau}_{n}\right) \hat{\tau}_{n}=R\left(t_{n}\right) \hat{\eta}_{n} \wedge \dot{x}\left(t_{n}^{+}\right)=C\left(t_{n}^{+}\right)
\end{aligned}
$$

hence the proposition is proved.

Since the motion is in the plane we have $C(t)=(0,0, c)$. If $c=0$, the motion is along a diameter and never leaves the diameter. Moreover, there is a symmetry between motion in the clockwise and in the anticlockwise direction given by changing sign to $c$ (see (3.1)). Without loss of generality in the following we assume $c>0$, which corresponds to anticlockwise motion.

Fixed the angular momentum $c$ and assuming that the function $R(t)$ is periodic, a bouncing solution can be obtained as the orbit of a map, the breathing circle billiard map, on a two-dimensional cylinder. Since $R(t)$ is not constant, the radial velocity of the particle has varying moduli so that a good choice of coordinates is given by the time of an impact and the radial velocity after an impact. Other velocity-related coordinates for the second component work as well. If $R \in C^{k}$ with $k>7$, it has been shown in [29] that the billiard map admits invariant circles which are homotopically non-trivial, thus showing that the velocity of a particle remains bounded. Their proof follows by showing that for high velocities the billiard map can be written as a small perturbation of an integrable map. Our main result in the case of highly regular functions $R$ may be read as a completion of that in [29], as we prove the coexistence of regular and of chaotic orbits in the phase space. However, our results hold under more general assumptions. If $R \in C^{2}$, we prove existence of both chaotic and regular motions. An informal statement is the following.

Main result. There exists a class of $C^{2}$, strictly positive and 1-periodic functions $\widetilde{\mathcal{R}}$, which contains the functions $R_{\delta, M}(t):=M+\delta \sin (2 \pi t)$ for $\delta \in$ $\left(\frac{1}{8 \pi^{2}}, 2 \pi\right)$ and $M$ large enough, such that if $R(t) \in \widetilde{\mathcal{R}}$ and $c$ is sufficiently small, then the breathing circle billiard map has positive topological entropy and admits periodic and quasi-periodic solutions. 
The formal statements along with the definitions of the class $\widetilde{\mathcal{R}}$ and of quasi-periodic bouncing solutions are given in Sect.2.1. We now discuss the geometric ideas underlying the proofs of this result.

Let us first consider the simple case in which $R(t) \equiv R^{*}>0$, that is the circle $\mathcal{D}$ is fixed. It is well known that in this case the norm of the velocity $v(t):=|\dot{x}(t)|$ of a solution and the angle $\varphi$ of reflection at $\partial \mathcal{D}$ are preserved at every bounce. Hence, the radial velocity of the particle is constant and a good set of coordinates for the billiard map is given by the arc length $s$ of $\partial \mathcal{D}$ and the angle $\varphi$. The motion is then described by the billiard map $B\left(s_{0}, \varphi_{0}\right)=\left(s_{1}, \varphi_{1}\right)$ of the cylinder $\mathbb{R} / 2 \pi R^{*} \mathbb{Z} \times\left(0, \frac{\pi}{2}\right)$ given by

$$
s_{1}=s_{0}+2 R^{*} \varphi_{0}, \quad \varphi_{1}=\varphi_{0} .
$$

The corresponding orbits $\left(s_{0}+2 R^{*} n \varphi_{0}, \varphi_{0}\right)_{n \in \mathbb{Z}}$ represent the successive impact points on the boundary and the respective angles of reflection. It is clear that a trajectory is uniquely determined (up to rotations) by the value $\varphi_{0}$, and introducing the rotation number $\omega=\pi \varphi_{0} \in\left(0, \frac{1}{2}\right)$ one finds that if $\omega=$ $p / q \in \mathbb{Q}$ then the trajectory closes after $q$ bounces and $p$ revolutions, while if $\omega \in \mathbb{R} \backslash \mathbb{Q}$ then the trajectory never closes and the bouncing points are dense on the boundary.

In addition, the map $B$ is exact symplectic in the sense that for all $\left(s_{0}, \varphi_{0}\right) \in \mathbb{R} / 2 \pi R^{*} \mathbb{Z} \times\left(0, \frac{\pi}{2}\right)$ the condition $\varphi_{1} d s_{1}-\varphi_{0} d s_{0}=d V\left(s_{0}, \varphi_{0}\right)$ is satisfied with $V\left(s_{0}, \varphi_{0}\right)=R^{*} \varphi_{0}^{2}$, and (positive) twist in the sense that for all $\left(s_{0}, \varphi_{0}\right) \in \mathbb{R} / 2 \pi R^{*} \mathbb{Z} \times\left(0, \frac{\pi}{2}\right)$

$$
\frac{\partial s_{1}}{\partial \varphi_{0}}>0
$$

This implies that it is implicitly given by means of a generating function $h$ : $\mathbb{R}^{2} \rightarrow \mathbb{R}^{2}$ given by $h\left(s_{0}, s_{1}\right)=\frac{1}{4 R^{*}}\left(s_{1}-s_{0}\right)^{2}$ through the equations

$$
\varphi_{0}=-\frac{\partial h}{\partial s_{0}}\left(s_{0}, s_{1}\right), \quad \varphi_{1}=\frac{\partial h}{\partial s_{1}}\left(s_{0}, s_{1}\right) .
$$

Finally, one observes that the (positive) twist condition is equivalent to

$$
\frac{\partial^{2} h}{\partial s_{1} \partial s_{0}}\left(s_{0}, s_{1}\right)<0
$$

for all $\left(s_{0}, s_{1}\right) \in \mathbb{R}^{2}$, which implies that (2.1) and (2.2) are equivalent. Exact symplectic (positive or negative) twist maps enjoy several properties that can be deduced only by the properties of the generating function and are at the basis of Aubry-Mather theory (see Appendix B).

In the first part of this paper, we follow the approach in [16] to find an exact symplectic twist map that describes the motion of some bouncing solutions of the breathing circle billiard. However, it turns out that the variables $(s, \varphi)$ are not a good choice to this aim, but as in [29] it is better to work with time and velocity. Again in the case of the fixed circle $\mathcal{D}$ with $R(t) \equiv R^{*}$, consider the variables $\left(t, v^{2}(t)\right)$ where $t$ represents the bouncing time. By Proposition 2.1, fixed the angular momentum $c>0$, we can consider 
the map $B_{c}\left(t_{0}, v_{0}^{2}\right)=\left(t_{1}, v_{1}^{2}\right)$ of the cylinder $\mathbb{R} / \mathbb{Z} \times \mathbb{R}^{+}$, depending on the parameter $c$, given by

$$
t_{1}=t_{0}+2 \frac{\sqrt{\left(R^{*}\right)^{2} v_{0}^{2}-c^{2}}}{v_{0}^{2}}=: t_{0}+f_{c}\left(v_{0}^{2}\right), \quad v_{1}^{2}=v_{0}^{2} .
$$

where $v_{i}^{2}:=v^{2}\left(t_{i}\right)$. Here, $f_{c}\left(v^{2}\right)$ plays the role of the rotation number. Notice that the map $B_{c}$ is still exact symplectic, since $v_{1}^{2} d t_{1}-v_{0}^{2} d t_{0}=d V\left(t_{0}, v_{0}^{2}\right)$ for $V\left(t_{0}, v_{0}^{2}\right)=v_{0}^{2} f_{c}\left(v_{0}^{2}\right)-F_{c}\left(v_{0}^{2}\right)$ where $F_{c}$ is a primitive of $f_{c}$. However, it does not satisfy the twist condition, as $f_{c}^{\prime}\left(v^{2}\right)$ has one change of sign. This has the following geometric interpretation: fixing $c$ there exist two possible trajectories joining two points on the boundary in time $t_{1}-t_{0}$. More precisely,

$$
f_{c}\left(v^{2}\right)=t_{1}-t_{0} \Longleftrightarrow v_{ \pm}^{2}\left(t_{1}-t_{0}\right)=\frac{2\left(R^{*}\right)^{2} \pm 2 \sqrt{\left(R^{*}\right)^{4}-c^{2}\left(t_{1}-t_{0}\right)^{2}}}{\left(t_{1}-t_{0}\right)^{2}}
$$

hence using $c=R^{*} v \cos \varphi$ one gets two values for the angles $\varphi_{ \pm}$of reflection.

This problem can be overcome by considering only motions with large values of $v^{2}$. Actually, it can be verified that for $v^{2}$ large, $B_{c}\left(t_{0}, v_{0}^{2}\right)$ satisfies the (negative) twist condition and can be expressed implicitly as in (2.2) by using the generating function

$$
h^{c}\left(t_{0}, t_{1}\right)=-\left(t_{1}-t_{0}\right) v_{+}^{2}\left(t_{1}-t_{0}\right)-2 c \arctan \left(\frac{c\left(t_{1}-t_{0}\right)}{\sqrt{\left(R^{*}\right)^{4}-c^{2}\left(t_{1}-t_{0}\right)^{2}}}\right) .
$$

Thus, one obtains an exact symplectic twist map, which describes the motion of some bouncing solutions of the fixed circle billiard.

The main idea behind the present paper is to replicate this last construction in the case of the breathing circle $\mathcal{D}_{t}$. Given a $C^{2}$, strictly positive and 1-periodic function $R(t)$ and fixed the angular momentum $c$, we show that there exist variables $(t, K)$ in the cylinder $\mathbb{R} / \mathbb{Z} \times \mathbb{R}^{+}$such that the billiard map $P(t, K)$ is exact symplectic and twist for $K$ large enough. The variable $K$ is a generalisation of $v^{2}$ and reduces to $\frac{v^{2}}{2}$ for $R(t)$ constant. This program is developed in Sects. 3 and 4 where the generating function is obtained by using the solutions of a suitable Dirichlet problem.

Thus, we show that Aubry-Mather theory can be applied to the exact symplectic twist map $P$, and the last part of the paper is devoted to proving the main result.

First of all, one gets the existence of interesting invariant sets, called Aubry-Mather sets, whose properties are summarised in Appendix B. AubryMather sets are characterised by their rotation number $\omega$; hence, we denote them by $M_{\omega}$. If $\omega=p / q \in \mathbb{Q}$; then, $M_{\omega}$ contains a periodic orbit, instead if $\omega \in \mathbb{R} \backslash \mathbb{Q}$ then $M_{\omega}$ is a graph over the first coordinate of the cylinder $\mathbb{R} / \mathbb{Z} \times \mathbb{R}^{+}$and is either an invariant curve which is homotopically non-trivial or a Cantor set. Orbits on $M_{\omega}$ give rise to interesting bouncing solutions of the breathing billiards: in the case $\omega=p / q \in \mathbb{Q}$ one gets periodic solutions of period $p$ with $q$ bounces in a period, while in the case $\omega \in \mathbb{R} \backslash \mathbb{Q}$ one gets families of quasi-periodic solutions of frequencies $(1,1 / \omega)$ (see Definition 2.4). 
By the properties of the billiard map $P$, we thus obtain existence of periodic and quasi-periodic orbits for some values of the rotation number $\omega$. In fact, since we can only guarantee that the map $P$ is exact symplectic and twist for large values of the coordinate $K$, we can apply Aubry-Mather theory to $P$ only for a subset of the possible rotation numbers and we need to assume that $R$ belongs to the class $\mathcal{R}$ defined in Definition 2.2. This is discussed in Sect. 5 where we give the proof of our first result, Theorem 2.5.

Then, Aubry-Mather theory gives also the tools to prove the existence of chaotic motion for exact symplectic twist maps on a cylinder. Namely it is well known (see Theorem B.6) that if an Aubry-Mather set of irrational rotation number is a Cantor set, then close to it there exist many invariant probability measures with positive metric entropy; hence, the map has positive topological entropy. Unfortunately the supports of these measures have zero Lebesgue measure in the cylinder, so that the problem of proving chaotic behaviour in the sense of smooth ergodic theory remains open.

In order to prove that an Aubry-Mather set with irrational rotation number is a Cantor set, as explained before, one needs to prove that it cannot be an invariant curve. Many techniques are available to prove the non-existence of invariant curves and form the so-called converse KAM theory. Here we are using the variational characterisation of the invariant curves for exact symplectic twist maps. We recall that there exists an action functional for which the orbits of an exact symplectic twist map are critical points, and in particular each orbit lying on an invariant curve is a point of minimum. As a consequence, the second variation of the action along an invariant curve is positive (see the function in Proposition B.7-(ii)). The basic idea to prove that a map does not have an invariant curve in a region of the cylinder is then to show that the second variation is negative in the whole region. We apply this method to the map $P$ as showed in Sect. 6. For us, it is enough to find a vertical segment $\ell$ in the cylinder on which the second variation of the action is negative, so that $\ell$ cannot be crossed by any invariant curve. Then we show that there exists a set $\mathcal{I} \subset \mathbb{R}$ such that the Aubry-Mather sets of the map $P$ with rotation number $\omega \in \mathcal{I}$ must cross the vertical segment $\ell$. This is achieved by recalling that the rotation number gives an estimate on the zone of the cylinder on which the corresponding Aubry-Mather set is located. In the end, we have showed that the Aubry-Mather sets $M_{\omega}$ with $\omega \in \mathcal{I}$ cannot be invariant curves, and this implies our main result stated in Theorem 2.7, namely that the map $P$ is chaotic in the sense that it has positive topological entropy. We remark that the proof of this result needs the assumption that the angular momentum $c$ is small enough and that the boundary of the circle $\mathcal{D}_{t}$ is described by a function in $\widetilde{\mathcal{R}} \subsetneq \mathcal{R}$. This last restriction is used to study the sign of the second variation of the action functional.

As a final comment, we remark that on one side the class $\widetilde{\mathcal{R}}$ contains "natural" choices of behaviours of the breathing circle such as the functions $R_{\delta, M}(t)$, which give rise to large circles with relatively small and slow breathing, thus intuitively describing a system which for large velocities is a small perturbation of the integrable system given by the billiard in a fixed circle. On 
the other side, we cannot say that the class $\widetilde{\mathcal{R}}$ is optimal to obtain the existence of chaotic motion for the breathing circle billiard. Its technical definition mainly follows from the methods of our proofs.

It is worth noting that the unperturbed map $B_{c}$ is exact and the twist has exactly one change of sign, so that it is a non-twist map as defined in $[7,8]$. However, it is not clear if also the billiard map $P$ is a non-twist map as we only prove that it can be expressed via a good generating function only for large values of the coordinate $K$.

\subsection{Formal Statement of the Main Result}

We split our main result into two theorems: Theorem 2.5 refers to periodic and quasi-periodic motions, while Theorem 2.7 concerns the chaotic behaviour.

Let us first introduce the classes of functions we consider for the motion of $\partial \mathcal{D}_{t}$.

Definition 2.2. Let $R(t)$ be a $C^{2}$, strictly positive and 1-periodic function, and $\varepsilon \in(0,1)$ be a fixed parameter. We denote by $\|\cdot\|$ the sup-norm of a function and use the notations

$$
\underline{R}:=\min _{t} R(t), \quad \bar{R}:=\max _{t} R(t)
$$

and

$$
\sigma:=\min \left\{\frac{\underline{R}}{2\|\dot{R}\|}, \frac{2 \sqrt{1+\sqrt{1-\varepsilon^{2}}} \underline{R}}{\sqrt{\left\|\frac{d^{2}}{d t^{2}} R^{2}\right\|}}\right\} .
$$

We say that $R(t)$ belongs to the class $\mathcal{R}$ if $\sigma>2$.

We say that $R(t)$ belongs to the class $\widetilde{\mathcal{R}}$ if:

(i) $\sigma>4$;

and there exists $\bar{t} \in[0,1)$ such that $(\ddot{R}(\bar{t}) \underline{R}+\|\dot{R}\| \bar{R})<0$ and

$$
3<1+\sqrt{\frac{2 \bar{R}^{2}}{-\ddot{R}(\bar{t}) \underline{R}-\|\dot{R}\| \bar{R}}}<-1+\sqrt{\frac{2 \underline{R}^{2}}{\frac{2 \bar{R}^{2}}{\sigma^{2}}+\|\dot{R}\| \bar{R}}} ;
$$

(iii)

$$
\dot{R}(\bar{t})=0 \quad \text { and } \quad \ddot{R}(\bar{t})<-\frac{2 \bar{R}^{2}}{\sigma^{2} \underline{R}} .
$$

For simplicity, we drop the dependence on $\varepsilon$ in the notations for $\sigma$ and the classes $\mathcal{R}$ and $\widetilde{\mathcal{R}}$. Clearly $\widetilde{\mathcal{R}} \subset \mathcal{R}$ and the classes $\mathcal{R}$ and $\widetilde{\mathcal{R}}$ are non-empty as shown by the next proposition whose technical proof is in Appendix A. In the following, the parameter $\varepsilon$ is to be considered fixed in $(0,1)$; there is not a more interesting value for it. However, notice that the class $\widetilde{\mathcal{R}}$ is empty if $\varepsilon=1$ (see Remark A.2). 
Proposition 2.3. The functions

$$
R_{\delta, M}(t):=M+\delta \sin (2 \pi t)
$$

with $\delta \in\left(\frac{1}{8 \pi^{2}}, 2 \pi\right)$ and $M$ large enough belong to the class $\widetilde{\mathcal{R}}$.

We now begin to state our main results. The first concerns the existence of regular motion for the billiard dynamics inside $\mathcal{D}_{t}$. We first give the definition of periodic and quasi-periodic bouncing solutions, inspired by [32] (see also $[30])$ :

Definition 2.4. Given $\omega \in \mathbb{R}$, we say that a family of bouncing solutions

$$
\left\{x_{\xi}(t)\right\}_{\xi \in \mathbb{R}}
$$

is a family of generalised quasi-periodic solutions of frequencies $(1,1 / \omega)$ if

(i) there exists a function $\Phi_{\xi}(a, b)$ such that

- the function $\xi \mapsto \Phi_{\xi}(a, b)$ is of bounded variation for all $(a, b)$, and if $\xi$ is a point of continuity, then so are $\xi \pm \omega$ and $\xi \pm 1$;

- $\Phi_{\xi}(a+1, b)=\Phi_{\xi}(a, b)=\Phi_{\xi}(a, b+1)$ for all $(a, b)$;

- for every $\xi \in \mathbb{R}$, the function $t \mapsto \Phi_{\xi}(t, t / \omega)$ is continuous;

$-x_{\xi}(t)=\Phi_{\xi}(t, t / \omega)$

(ii) for every $\xi \in \mathbb{R}$, the sequence of impact times $\left(t_{n}\right)_{n \in \mathbb{Z}}$ satisfies

$$
\lim _{n \rightarrow \infty} \frac{t_{n}}{n}=\omega
$$

and if $\omega=p / q \in \mathbb{Q}$ then $t_{n+q}=t_{n}+p$ for every $n \in \mathbb{Z}$.

If $\omega \in \mathbb{R} \backslash \mathbb{Q}$ and the function $(\xi, a, b) \mapsto \Phi_{\xi}(a, b)$ is continuous, then these solutions are called classical quasi-periodic.

If $\omega=p / q \in \mathbb{Q}$, then for every $\xi \in \mathbb{R}$

$$
x_{\xi}(t+p)=\Phi_{\xi}\left(t+p, \frac{q}{p} t+q\right)=\Phi_{\xi}\left(t, \frac{q}{p} t\right)=x_{\xi}(t)
$$

so that the bouncing solution makes $q$ bounces in time $p$ before repeating itself. They are said $(p, q)$-periodic.

Theorem 2.5. Suppose that $c \in\left(0, \varepsilon \frac{\underline{R}^{2}}{\sigma}\right)$ and let $R(t) \in \mathcal{R}$. Then, for every $1<\omega<\sigma-1$ there exists a family of generalised quasi-periodic bouncing solutions of frequencies $(1,1 / \omega)$.

Remark 2.6. Under the hypotheses of Theorem 2.5 we are not able to guarantee that the solutions are classical quasi-periodic. Moreover, the restriction $\omega>1$ is not optimal and is due to the techniques used in the proof.

Let us now consider the existence of chaotic dynamics for the billiard motion in $\mathcal{D}_{t}$. Let $P$ be the breathing circle billiard map as defined in Proposition 4.4 .

Theorem 2.7. Suppose that $R(t) \in \widetilde{\mathcal{R}}$. Then, for c sufficiently small, the breathing circle billiard map $P$ has positive topological entropy. 
The result is proved in Sect. 6 . It follows by a standard application of the Variational Principle, showing the existence of invariant probability measures with positive metric entropy. Such measures are obtained by applying results in [11]. We remark that the union of the supports of these invariant measures has zero Lebesgue measure. However, the existence of invariant probability measures with positive metric entropy and support of positive Lebesgue measure is a major problem in dynamical systems and is an open problem also for well-known twist maps of the cylinder as the standard map.

Remark 2.8. We have stated Theorems 2.5 and 2.7 for positive values of the angular momentum $c$, the case $c=0$ corresponding to motions along a diameter and the system reducing to the Fermi-Ulam model. Our arguments can be readily adapted to the case $c=0$, so that the conclusions of our main theorems also hold for the Fermi-Ulam model.

\section{The Dirichlet Problem}

The proofs of our main results rely on Aubry-Mather theory, for which we need to define a generating function for the billiard map associated with the billiard flow in $\mathcal{D}_{t}$. The first step is the study of the Dirichlet problem associated with the flow between two consecutive bounces.

Let us consider two consecutive bounces for the billiard motion in $\mathcal{D}_{t}$ at times $t_{n}$ and $t_{n+1}$; the Dirichlet problem which describes the billiard motion between the two bounces is

$$
\left\{\begin{array}{l}
\ddot{x}(t)=0, \quad t \in\left(t_{n}, t_{n+1}\right), \\
|x(t)|<R(t), \quad t \in\left(t_{n}, t_{n+1}\right), \\
\left|x\left(t_{n}\right)\right|=R\left(t_{n}\right), \\
\left|x\left(t_{n+1}\right)\right|=R\left(t_{n+1}\right)
\end{array}\right.
$$

and in polar coordinates $(r, \theta)$ it transforms into

$$
\left\{\begin{array}{l}
\ddot{r}=\frac{c^{2}}{r^{3}}, \quad t \in\left(t_{n}, t_{n+1}\right) \\
r^{2} \dot{\theta}=c, \quad t \in\left(t_{n}, t_{n+1}\right) \\
r(t)<R(t), \quad t \in\left(t_{n}, t_{n+1}\right) \\
r\left(t_{n}\right)=R\left(t_{n}\right) \\
r\left(t_{n+1}\right)=R\left(t_{n+1}\right)
\end{array}\right.
$$

from which it is evident the symmetry with respect to the change of $\operatorname{sign}$ of $c$. Bouncing condition 4 in these coordinates reads

$$
\begin{aligned}
& \dot{\theta}\left(t_{n}^{+}\right)=\dot{\theta}\left(t_{n}^{-}\right), \\
& \dot{r}\left(t_{n}^{+}\right)=-\dot{r}\left(t_{n}^{-}\right)+2 \dot{R}\left(t_{n}\right) .
\end{aligned}
$$

In the following result, we find sufficient conditions for a solution of system (3.1) to exist. Note that these solutions do not satisfy in general the bouncing conditions (3.2)-(3.3) when glued together. 
Proposition 3.1. Let $\varepsilon \in(0,1)$ be a fixed parameter, and let us fix a value of $c>0$. For all times $t_{n}, t_{n+1}$ satisfying

$$
\begin{aligned}
& 0<t_{n+1}-t_{n}<\varepsilon \frac{\underline{R}^{2}}{c}, \\
& 0<t_{n+1}-t_{n}<\frac{\underline{R}}{2\|\dot{R}\|}, \\
& 0<t_{n+1}-t_{n}<\frac{2 \sqrt{1+\sqrt{1-\varepsilon^{2}}} \underline{R}}{\sqrt{\left\|\frac{d^{2}}{d t^{2}} R^{2}\right\|}}
\end{aligned}
$$

system (3.1) admits a unique $C^{2}$ solution $\left(r\left(t ; t_{n}, t_{n+1}\right), \theta\left(t ; t_{n}, t_{n+1}\right)\right)$ such that

$$
\dot{r}\left(t_{n}^{+}\right)<-\frac{R\left(t_{n}\right)}{t_{n+1}-t_{n}}, \quad \dot{r}\left(t_{n+1}^{-}\right)>\frac{R\left(t_{n+1}\right)}{t_{n+1}-t_{n}},
$$

and

$$
\dot{r}\left(t_{n}^{+}\right)<\dot{R}\left(t_{n}\right), \quad \dot{r}\left(t_{n+1}^{-}\right)>2 \dot{R}\left(t_{n+1}\right) .
$$

Proof. Define $R_{n}=R\left(t_{n}\right), R_{n+1}=R\left(t_{n+1}\right)$ and $\tau_{n}=t_{n+1}-t_{n}$. The equation $\ddot{r}=c^{2} / r^{3}$ has the first integral

$$
A=2 E=\dot{r}^{2}+\frac{c^{2}}{r^{2}}>0
$$

where $E$ is the energy of the system, and can be integrated giving the general solution

$$
r(t)=\sqrt{\frac{c^{2}+A^{2}(t+B)^{2}}{A}}
$$

for $B \in \mathbb{R}$. System (3.1) is rotationally invariant so that we can fix $\theta\left(t_{n}\right)=0$ without loss of generality. With this assumption, solution (3.10) represents a straight trajectory in the billiard table starting from the point $\left(R_{n}, 0\right)$ with velocity $\dot{x}\left(t_{n}\right)$ satisfying

$$
\dot{x}\left(t_{n}\right)=\left(\begin{array}{c}
\frac{A\left(t_{n}+B\right)}{R_{n}} \\
\frac{c}{R_{n}}
\end{array}\right) \quad \text { and } \quad\left|\dot{x}\left(t_{n}\right)\right|^{2}=2 E=A .
$$

Moreover, notice that by $(3.10)$

$$
\dot{r}(t)=\frac{A(t+B)}{r(t)} .
$$

Let $\ell:=A\left(t_{n}+B\right)$, then the straight trajectory (3.10) is parametrised by

$$
[0,+\infty) \ni s \mapsto\left(\begin{array}{c}
R_{n}+s \frac{\ell}{R_{n}} \\
s \frac{c}{R_{n}}
\end{array}\right)
$$

and the condition $r\left(t_{n+1}\right)=R_{n+1}$ is then equivalent to

$$
\left(R_{n}+\tau_{n} \frac{\ell}{R_{n}}\right)^{2}+\tau_{n}^{2} \frac{c^{2}}{R_{n}^{2}}=R_{n+1}^{2} .
$$


We then obtain

$$
\ell_{ \pm}=\frac{R_{n}}{\tau_{n}}\left(-R_{n} \pm \sqrt{R_{n+1}^{2}-\tau_{n}^{2} \frac{c^{2}}{R_{n}^{2}}}\right)
$$

which is well defined thanks to (3.4). Using (3.11), it also holds

$$
A_{ \pm}=\frac{\ell_{ \pm}^{2}+c^{2}}{R_{n}^{2}}, \quad B_{ \pm}=\frac{R_{n}^{2} \ell_{ \pm}}{\ell_{ \pm}^{2}+c^{2}}
$$

which in (3.10) gives $r\left(t_{n}\right)=R_{n}$. We still have to verify conditions (3.7), (3.8) and $r(t)<R(t)$ for all $t \in\left(t_{n}, t_{n+1}\right)$.

Using (3.12), the first condition in (3.7) reduces to

$$
\dot{r}\left(t_{n}^{+}\right)=\frac{\ell_{ \pm}}{R_{n}}<-\frac{R_{n}}{\tau_{n}} .
$$

From (3.13), it is immediate that the choice $\ell_{+}$has to be discarded and $\ell_{-}$is admissible. Let us now consider the second condition in (3.7). From (3.12), we need to check that

$$
\dot{r}\left(t_{n+1}^{-}\right)=\frac{A\left(t_{n+1}+B\right)}{R_{n+1}}=\frac{A \tau_{n}+\ell_{-}}{R_{n+1}}>\frac{R_{n+1}}{\tau_{n}} .
$$

But, by (3.14) we have

$$
\begin{aligned}
A \tau_{n}+\ell_{-} & =\frac{\ell_{-}^{2}+c^{2}}{R_{n}^{2}} \tau_{n}+\ell_{-} \\
& =\frac{1}{\tau_{n}}\left[\left(R_{n}+\sqrt{R_{n+1}^{2}-\tau_{n}^{2} \frac{c^{2}}{R_{n}^{2}}}\right)^{2}+\tau_{n}^{2} \frac{c^{2}}{R_{n}^{2}}-R_{n}\left(R_{n}+\sqrt{R_{n+1}^{2}-\tau_{n}^{2} \frac{c^{2}}{R_{n}^{2}}}\right)\right] \\
& =\frac{1}{\tau_{n}}\left(R_{n+1}^{2}+R_{n} \sqrt{R_{n+1}^{2}-\tau_{n}^{2} \frac{c^{2}}{R_{n}^{2}}}\right)>\frac{R_{n+1}^{2}}{\tau_{n}},
\end{aligned}
$$

so that both conditions (3.7) are satisfied only for $\ell_{-}$. Moreover, from (3.5),

$$
\left|\dot{R}\left(t_{n}\right)\right| \leq 2\|\dot{R}\|<\frac{\underline{R}}{\tau_{n}} \leq \frac{R_{n}}{\tau_{n}},
$$

from which, using the first condition in (3.7), we get

$$
\dot{r}\left(t_{n}^{+}\right)<-\frac{R_{n}}{\tau_{n}}<\dot{R}\left(t_{n}\right)
$$

that is the first condition in (3.8). The second condition is proved similarly.

It remains to show that $r(t)<R(t)$ for all $t \in\left(t_{n}, t_{n+1}\right)$. From (3.8), it follows that

$$
\left.\frac{\mathrm{d}}{\mathrm{d} t}\left(R^{2}(t)-r^{2}(t)\right)\right|_{t=t_{n}^{+}}>0,\left.\quad \frac{\mathrm{d}}{\mathrm{d} t}\left(R^{2}(t)-r^{2}(t)\right)\right|_{t=t_{n+1}^{-}}<0 .
$$

Moreover from (3.12) and (3.14) 


$$
\begin{aligned}
& \frac{\mathrm{d}^{2}}{\mathrm{~d} t^{2}}\left(R^{2}(t)-r^{2}(t)\right)=\left(\frac{\mathrm{d}^{2}}{\mathrm{~d} t^{2}} R^{2}(t)\right)-2 A= \\
& \quad=\left(\frac{\mathrm{d}^{2}}{\mathrm{~d} t^{2}} R^{2}(t)\right)-\frac{2}{\tau_{n}^{2}}\left(R_{n}^{2}+R_{n+1}^{2}+2 R_{n} \sqrt{R_{n+1}^{2}-\tau_{n}^{2} \frac{c^{2}}{R_{n}^{2}}}\right)< \\
& \quad<\left\|\frac{\mathrm{d}^{2}}{\mathrm{~d} t^{2}} R^{2}\right\|-\frac{2}{\tau_{n}^{2}}\left(2 \underline{R}^{2}+2 \underline{R} \sqrt{\underline{R}^{2}\left(1-\varepsilon^{2}\right)}\right)
\end{aligned}
$$

where in the last inequality we have used (3.4). It follows that

$$
\frac{\mathrm{d}^{2}}{\mathrm{~d} t^{2}}\left(R^{2}(t)-r^{2}(t)\right)<\left\|\frac{\mathrm{d}^{2}}{\mathrm{~d} t^{2}} R^{2}\right\|-\frac{4\left(1+\sqrt{1-\varepsilon^{2}}\right) \underline{R}^{2}}{\tau_{n}^{2}}<0
$$

by (3.6). Hence, since $r^{2}\left(t_{n}\right)=R^{2}\left(t_{n}\right)$ and $r^{2}\left(t_{n+1}\right)=R^{2}\left(t_{n+1}\right)$, we have that $R^{2}(t)>r^{2}(t)$ for all $t \in\left(t_{n}, t_{n+1}\right)$. This concludes the proof of the proposition, for which the solution is given by (3.10) with $A, B$ as in (3.14) with $\ell=\ell_{-}$as defined in (3.13).

In conclusion, we have proved that for all times $t_{n}, t_{n+1}$ satisfying (3.4)(3.6), the functions

$$
\begin{aligned}
& r\left(t ; t_{n}, t_{n+1}\right):=\sqrt{\frac{A^{2}\left(t_{n}, t_{n+1}\right)\left(t+B\left(t_{n}, t_{n+1}\right)\right)^{2}+c^{2}}{A\left(t_{n}, t_{n+1}\right)},} \\
& \theta\left(t ; t_{n}, t_{n+1}\right):=\theta\left(t_{n}\right)+\int_{t_{n}}^{t_{n+1}} \frac{c}{r^{2}\left(t ; t_{n}, t_{n+1}\right)} \mathrm{d} t
\end{aligned}
$$

where $\theta\left(t_{n}\right)$ is the angular variable of the bounce point at time $t_{n}$, are a solution to system (3.1) satisfying also conditions (3.7)-(3.8) by letting

$$
\begin{aligned}
A\left(t_{n}, t_{n+1}\right) & \\
= & \frac{R^{2}\left(t_{n}\right)+R^{2}\left(t_{n+1}\right)+2 \sqrt{R^{2}\left(t_{n}\right) R^{2}\left(t_{n+1}\right)-c^{2}\left(t_{n+1}-t_{n}\right)^{2}}}{\left(t_{n+1}-t_{n}\right)^{2}}, \\
& B\left(t_{n}, t_{n+1}\right) \\
= & -\left(t_{n}+\frac{R^{2}\left(t_{n}\right)+\sqrt{R^{2}\left(t_{n}\right) R^{2}\left(t_{n+1}\right)-c^{2}\left(t_{n+1}-t_{n}\right)^{2}}}{\left(t_{n+1}-t_{n}\right) A\left(t_{n}, t_{n+1}\right)}\right) .
\end{aligned}
$$

The $C^{2}$ regularity of the solution $\left(r\left(t ; t_{n}, t_{n+1}\right), \theta\left(t ; t_{n}, t_{n+1}\right)\right)$ with respect to all its variables follows from (3.15)-(3.16).

Remark 3.2. Given times $t_{n}, t_{n+1}$ and $t_{n+2}$ such that the intervals $\left(t_{n+1}-t_{n}\right)$ and $\left(t_{n+2}-t_{n+1}\right)$ satisfy assumptions (3.4)-(3.6), the solutions $r\left(t ; t_{n}, t_{n+1}\right)$ and $r\left(t ; t_{n+1}, t_{n+2}\right)$ given in Proposition 3.1 in general do not satisfy the bouncing condition (3.3) at time $t_{n+1}$. However, in Sect. 4 we find a method to get sequences $\left(t_{n}\right)_{n \in \mathbb{Z}}$ satisfying conditions (3.4)-(3.6) for every $n$ and such that the corresponding functions defined in (3.15) satisfy the bouncing condition (3.3). Hence, we will construct bouncing solutions satisfying conditions (3.7)(3.8) at every bouncing time. 
Remark 3.3. Given $\theta\left(t_{n}\right)$ and $\theta\left(t_{n+1}\right)$, the angular variables of two consecutive bounces at times $t_{n}$ and $t_{n+1}$ determined by solution (3.15), the angular variation $\delta \theta\left(t_{n}, t_{n+1}\right):=\theta\left(t_{n+1}\right)-\theta\left(t_{n}\right)$ is given by

$$
\delta \theta\left(t_{n}, t_{n+1}\right)=\pi-\arctan \left(\frac{c\left(t_{n+1}-t_{n}\right)}{\sqrt{R^{2}\left(t_{n}\right) R^{2}\left(t_{n+1}\right)-c^{2}\left(t_{n+1}-t_{n}\right)^{2}}}\right) .
$$

Indeed, the length of the trajectory between the two bounces is given by $\left(t_{n+1}-\right.$ $\left.t_{n}\right)\left|\dot{x}\left(t_{n}\right)\right|=\left(t_{n+1}-t_{n}\right) \sqrt{A\left(t_{n}, t_{n+1}\right)}$ (see $\left.(3.11)\right)$, and hence, by Carnot's theorem

$\left(t_{n+1}-t_{n}\right)^{2} A\left(t_{n}, t_{n+1}\right)=R^{2}\left(t_{n}\right)+R^{2}\left(t_{n+1}\right)-2 R\left(t_{n}\right) R\left(t_{n+1}\right) \cos \delta \theta\left(t_{n}, t_{n+1}\right)$ and by $(3.16)$

$$
\cos \delta \theta\left(t_{n}, t_{n+1}\right)=-\sqrt{1-\frac{c^{2}\left(t_{n+1}-t_{n}\right)^{2}}{R^{2}\left(t_{n}\right) R^{2}\left(t_{n+1}\right)}} .
$$

Then (3.17) immediately follows by computing $\sin \delta \theta\left(t_{n}, t_{n+1}\right)$ using that $c>$ 0 , so that $\delta \theta\left(t_{n}, t_{n+1}\right) \in(0, \pi)$.

\section{The Generating Function}

In this section, we define a generating function $h\left(t_{0}, t_{1}\right)$ for the billiard map between two consecutive bounces on $\partial \mathcal{D}_{t}$ at times $t_{0}$ and $t_{1}$. The orbits of the corresponding billiard map generate bouncing motions with large velocity, in the sense that conditions (3.7), (3.8) are satisfied.

Following the approach in [16], we first show that a good guess for $h$ is given by the action of system (3.1). Let

$$
h^{c}\left(t_{n}, t_{n+1}\right)=\int_{t_{n}}^{t_{n+1}} L^{c}\left(r\left(t ; t_{n}, t_{n+1}\right), \dot{r}\left(t ; t_{n}, t_{n+1}\right)\right) \mathrm{d} t
$$

where $r\left(t ; t_{n}, t_{n+1}\right)$ is the solution to (3.1) found in Proposition 3.1 and

$$
L^{c}(r, \dot{r})=\frac{1}{2} \dot{r}^{2}-\frac{c^{2}}{2 r^{2}}
$$

is the reduced Lagrangian of the system for a fixed value $c>0$. In particular, $r\left(t ; t_{n}, t_{n+1}\right)$ satisfies the Euler-Lagrange equation

$$
\frac{\mathrm{d}}{\mathrm{d} t}\left(\frac{\partial L^{c}}{\partial \dot{r}}\right)=\frac{\partial L^{c}}{\partial r} .
$$

For simplicity, let us denote $r(t)=r\left(t ; t_{n}, t_{n+1}\right)$ and remove the dependence on $c$. We have

$$
\begin{gathered}
\partial_{t_{n}} h\left(t_{n}, t_{n+1}\right)=\partial_{t_{n}} \int_{t_{n}}^{t_{n+1}} L(r(t), \dot{r}(t)) \mathrm{d} t=-L\left(r\left(t_{n}\right), \dot{r}\left(t_{n}^{+}\right)\right) \\
+\int_{t_{n}}^{t_{n+1}} \frac{\partial L}{\partial \dot{r}} \frac{\partial \dot{r}}{\partial t_{n}}+\frac{\partial L}{\partial r} \frac{\partial r}{\partial t_{n}} \mathrm{~d} t
\end{gathered}
$$




$$
\begin{aligned}
& =-L\left(r\left(t_{n}\right), \dot{r}\left(t_{n}^{+}\right)\right)+\left[\frac{\partial L}{\partial \dot{r}} \frac{\partial r}{\partial t_{n}}\right]_{t=t_{n}}^{t=t_{n+1}}+\int_{t_{n}}^{t_{n+1}}\left[-\frac{\mathrm{d}}{\mathrm{d} t}\left(\frac{\partial L}{\partial \dot{r}}\right)+\frac{\partial L}{\partial r}\right] \frac{\partial r}{\partial t_{n}} \mathrm{~d} t \\
& =-L\left(r\left(t_{n}\right), \dot{r}\left(t_{n}^{+}\right)\right)+\frac{\partial L}{\partial \dot{r}}\left(t_{n+1}\right) \frac{\partial r}{\partial t_{n}}\left(t_{n+1}\right)-\frac{\partial L}{\partial \dot{r}}\left(t_{n}\right) \frac{\partial r}{\partial t_{n}}\left(t_{n}\right),
\end{aligned}
$$

where we first used integration by parts, and then, the fact that $r(t)$ satisfies the Euler-Lagrange equation and finally denoted

$$
\frac{\partial L}{\partial \dot{r}}\left(t_{n+1}\right)=\frac{\partial L}{\partial \dot{r}}\left(r\left(t_{n+1}\right), \dot{r}\left(t_{n+1}^{+}\right)\right),
$$

and analogously for $\frac{\partial L}{\partial \dot{r}}\left(t_{n}\right)$. Differentiating with respect to $t_{n}$ the relations $r\left(t_{n+1} ; t_{n}, t_{n+1}\right)=R\left(t_{n+1}\right)$ and $r\left(t_{n} ; t_{n}, t_{n+1}\right)=R\left(t_{n}\right)$, we get

$$
\frac{\partial r}{\partial t_{n}}\left(t_{n+1}\right)=0, \quad \dot{r}\left(t_{n}^{+}\right)+\frac{\partial r}{\partial t_{n}}\left(t_{n}\right)=\dot{R}\left(t_{n}\right) .
$$

Hence, we have

$$
\partial_{t_{n}} h\left(t_{n}, t_{n+1}\right)=-L\left(r\left(t_{n}\right), \dot{r}\left(t_{n}^{+}\right)\right)+\frac{\partial L}{\partial \dot{r}}\left(t_{n}\right)\left(\dot{r}\left(t_{n}^{+}\right)-\dot{R}\left(t_{n}\right)\right)
$$

and, remembering the expression of $L$ in (4.2) and that $r\left(t_{n}\right)=R\left(t_{n}\right)$,

$$
\partial_{t_{n}} h\left(t_{n}, t_{n+1}\right)=\frac{1}{2} \dot{r}^{2}\left(t_{n}^{+}\right)+\frac{c^{2}}{2 R^{2}\left(t_{n}\right)}-\dot{r}\left(t_{n}^{+}\right) \dot{R}\left(t_{n}\right) .
$$

Analogously, one can get

$$
\partial_{t_{n+1}} h\left(t_{n}, t_{n+1}\right)=-\frac{1}{2} \dot{r}^{2}\left(t_{n+1}^{-}\right)-\frac{c^{2}}{2 R^{2}\left(t_{n+1}\right)}+\dot{r}\left(t_{n+1}^{-}\right) \dot{R}\left(t_{n+1}\right) .
$$

Therefore, we conclude that if a sequence $\left(t_{n}\right)$ satisfies

$$
\partial_{1} h\left(t_{n}, t_{n+1}\right)+\partial_{2} h\left(t_{n-1}, t_{n}\right)=0 \text { for every } n \in \mathbb{Z},
$$

where $\partial_{1}$ and $\partial_{2}$ denote differentiation with respect to the first and the second argument, respectively, then

$$
\begin{aligned}
\frac{1}{2} \dot{r}^{2}\left(t_{n}^{+}\right)-\dot{r}\left(t_{n}^{+}\right) \dot{R}\left(t_{n}\right) & =\frac{1}{2} \dot{r}^{2}\left(t_{n}^{-}\right)-\dot{r}\left(t_{n}^{-}\right) \dot{R}\left(t_{n}\right) \Leftrightarrow \\
\frac{1}{2} \dot{r}^{2}\left(t_{n}^{+}\right)-\dot{r}\left(t_{n}^{+}\right) \dot{R}\left(t_{n}\right)+\frac{1}{2} \dot{R}^{2}\left(t_{n}\right) & =\frac{1}{2} \dot{r}^{2}\left(t_{n}^{-}\right)-\dot{r}\left(t_{n}^{-}\right) \dot{R}\left(t_{n}\right)+\frac{1}{2} \dot{R}^{2}\left(t_{n}\right) \Leftrightarrow \\
\frac{1}{2}\left(\dot{r}\left(t_{n}^{+}\right)-\dot{R}\left(t_{n}\right)\right)^{2} & =\frac{1}{2}\left(\dot{r}\left(t_{n}^{-}\right)-\dot{R}\left(t_{n}\right)\right)^{2}
\end{aligned}
$$

from which we get the bouncing condition (3.3) using that $\dot{r}\left(t_{n}^{+}\right)<\dot{R}\left(t_{n}\right)$ and $\dot{r}\left(t_{n}^{-}\right)>\dot{R}\left(t_{n}\right)$ (see (3.8)). Conversely, a sequence satisfying (3.3) also satisfies (4.4). Using also Remark 3.2, we have proved the following

Proposition 4.1. A sequence $\left(t_{n}, \dot{r}\left(t_{n}^{+} ; t_{n}, t_{n+1}\right)\right)$, with $\left(t_{n+1}-t_{n}\right)$ satisfying (3.4)-(3.6) and $r\left(t ; t_{n}, t_{n+1}\right)$ being the solution to (3.1) given by Proposition 3.1 defines a bouncing solution with angular momentum $c$ if and only if for every $n \in \mathbb{Z}$

$$
\partial_{1} h^{c}\left(t_{n}, t_{n+1}\right)+\partial_{2} h^{c}\left(t_{n-1}, t_{n}\right)=0
$$

for the function $h^{c}\left(t_{n}, t_{n+1}\right)$ defined in (4.1). 
We are now ready to give an explicit expression to the function $h^{c}$ in terms of the times of bouncing.

Proposition 4.2. Fixed a value $c>0$ for the angular momentum, let $t_{0}, t_{1}$ be two consecutive bouncing times and $R_{0}:=R\left(t_{0}\right)$ and $R_{1}:=R\left(t_{1}\right)$ be the corresponding radii of the breathing circle. The generating function defined in (4.1) computed along solutions to (3.1) defined in (3.15) has the form

$$
h\left(t_{0}, t_{1}\right)=\frac{1}{2}\left(t_{1}-t_{0}\right) A\left(t_{0}, t_{1}\right)+c \arctan \left(\frac{c\left(t_{1}-t_{0}\right)}{\sqrt{R_{0}^{2} R_{1}^{2}-c^{2}\left(t_{1}-t_{0}\right)^{2}}}\right)
$$

where $A\left(t_{0}, t_{1}\right)$ is defined in (3.16).

Proof. Using (3.9) for solutions to (3.1) it holds

$$
L^{c}(r, \dot{r})=\frac{1}{2} \dot{r}^{2}-\frac{c^{2}}{2 r^{2}}=E-\frac{c^{2}}{2 r^{2}}=\frac{A\left(t_{0}, t_{1}\right)}{2}-\frac{c^{2}}{r^{2}} .
$$

Hence

$$
h^{c}\left(t_{0}, t_{1}\right)=\int_{t_{0}}^{t_{1}} L^{c}(r, \dot{r}) d t=\frac{1}{2}\left(t_{1}-t_{0}\right) A\left(t_{0}, t_{1}\right)-c \int_{t_{0}}^{t_{1}} \frac{c}{r^{2}\left(t ; t_{0}, t_{1}\right)} d t .
$$

Moreover by (3.15) for $\theta\left(t ; t_{0}, t_{1}\right)$ and Remark 3.3, we have

$$
\int_{t_{0}}^{t_{1}} \frac{c}{r^{2}\left(t ; t_{0}, t_{1}\right)} d t=\delta \theta\left(t_{0}, t_{1}\right)=\pi-\arctan \left(\frac{c\left(t_{1}-t_{0}\right)}{\sqrt{R_{0}^{2} R_{1}^{2}-c^{2}\left(t_{1}-t_{0}\right)^{2}}}\right)
$$

and the proof is finished, since the generating function is defined up to an additive constant.

We now argue on the other direction. Given the function $h\left(t_{0}, t_{1}\right)$ in (4.5), we show that when restricted to a suitable subset, it is a generating function of the bouncing motion of a particle inside the breathing circle $\mathcal{D}_{t}$.

Proposition 4.3. Let $\varepsilon \in(0,1)$ be a fixed parameter and let

$$
\sigma:=\min \left\{\frac{\underline{R}}{2\|\dot{R}\|}, \frac{2 \sqrt{1+\sqrt{1-\varepsilon^{2}}} \underline{R}}{\sqrt{\left\|\frac{d^{2}}{d t^{2}} R^{2}\right\|}}\right\} \quad \text { and } \quad c \in\left(0, \varepsilon \frac{\underline{R}^{2}}{\sigma}\right) .
$$

Let us consider the strip $\Omega=\left\{\left(t_{0}, t_{1}\right) \in \mathbb{R}^{2}: 0<t_{1}-t_{0}<\sigma\right\}$. Then, the function $h: \Omega \rightarrow \mathbb{R}$ defined in (4.5) satisfies

(i) $h \in C^{2}(\Omega)$;

(ii) $h\left(t_{0}+1, t_{1}+1\right)=h\left(t_{0}, t_{1}\right)$ for all $\left(t_{0}, t_{1}\right) \in \Omega$;

(iii) $\partial_{t_{0} t_{1}} h\left(t_{0}, t_{1}\right)<0$ for all $\left(t_{0}, t_{1}\right) \in \Omega$, and $\partial_{t_{0} t_{1}} h\left(t_{0}, t_{1}\right) \rightarrow-\infty$ as $\left(t_{1}-\right.$ $\left.t_{0}\right) \rightarrow 0$.

Proof. Properties (i) and (ii) follow immediately from the regularity and the periodicity of $R(t)$, and from the definition of $h\left(t_{0}, t_{1}\right)$.

To prove (iii), by standard computations we obtain

$$
\partial_{t_{0}} h\left(t_{0}, t_{1}\right)=\frac{c^{2}}{2 R_{0}^{2}}+\frac{1}{2}\left(\frac{R_{0}^{2}+\sqrt{R_{0}^{2} R_{1}^{2}-c^{2}\left(t_{1}-t_{0}\right)^{2}}}{R_{0}\left(t_{1}-t_{0}\right)}\right)^{2}
$$




$$
+\dot{R}\left(t_{0}\right) \frac{R_{0}^{2}+\sqrt{R_{0}^{2} R_{1}^{2}-c^{2}\left(t_{1}-t_{0}\right)^{2}}}{R_{0}\left(t_{1}-t_{0}\right)}
$$

hence, using that $h \in C^{2}$,

$$
\begin{aligned}
\partial_{t_{0} t_{1}} h\left(t_{0}, t_{1}\right)= & \left(\frac{R_{0}^{2}+\sqrt{R_{0}^{2} R_{1}^{2}-c^{2}\left(t_{1}-t_{0}\right)^{2}}}{R_{0}\left(t_{1}-t_{0}\right)}+\dot{R}\left(t_{0}\right)\right) \\
& \partial_{t_{1}}\left(\frac{R_{0}^{2}+\sqrt{R_{0}^{2} R_{1}^{2}-c^{2}\left(t_{1}-t_{0}\right)^{2}}}{R_{0}\left(t_{1}-t_{0}\right)}\right) .
\end{aligned}
$$

We can then conclude by the following estimates. First, if $\dot{R}\left(t_{0}\right) \geq 0$, then

$$
\frac{R_{0}^{2}+\sqrt{R_{0}^{2} R_{1}^{2}-c^{2}\left(t_{1}-t_{0}\right)^{2}}}{R_{0}\left(t_{1}-t_{0}\right)}+\dot{R}\left(t_{0}\right)>0 .
$$

If instead $\dot{R}\left(t_{0}\right)<0$, then we use $\left(t_{1}-t_{0}\right)<\sigma$ and write

$$
\frac{R_{0}^{2}+\sqrt{R_{0}^{2} R_{1}^{2}-c^{2}\left(t_{1}-t_{0}\right)^{2}}}{R_{0}\left(t_{1}-t_{0}\right)} \geq \frac{R_{0}}{t_{1}-t_{0}}>\frac{2\|\dot{R}\| R_{0}}{\underline{R}}>-\dot{R}\left(t_{0}\right)
$$

so that the first term in $\partial_{t_{0} t_{1}} h\left(t_{0}, t_{1}\right)$ is positive in all cases.

For the second term, we have

$$
\begin{aligned}
\partial_{t_{1}} & \left(\frac{R_{0}^{2}+\sqrt{R_{0}^{2} R_{1}^{2}-c^{2}\left(t_{1}-t_{0}\right)^{2}}}{R_{0}\left(t_{1}-t_{0}\right)}\right) \\
= & R_{0}^{2} \frac{R_{1} \dot{R}\left(t_{1}\right)\left(t_{1}-t_{0}\right)-\sqrt{R_{0}^{2} R_{1}^{2}-c^{2}\left(t_{1}-t_{0}\right)^{2}}-R_{1}^{2}}{\left(t_{1}-t_{0}\right)^{2} \sqrt{R_{0}^{2} R_{1}^{2}-c^{2}\left(t_{1}-t_{0}\right)^{2}}}
\end{aligned}
$$

which is negative both if $\dot{R}\left(t_{1}\right) \leq 0$, as sum of non-positive terms with $R_{1}>0$, and if $\dot{R}\left(t_{1}\right)>0$ since by $\left(t_{1}-t_{0}\right)<\sigma$ it holds

$$
R_{1} \dot{R}\left(t_{1}\right)\left(t_{1}-t_{0}\right)<\frac{R_{1} \dot{R}\left(t_{1}\right) \underline{R}}{2\|\dot{R}\|} \leq \frac{R_{1}^{2}}{2} .
$$

Finally, from (4.7) we find

$$
\partial_{t_{0} t_{1}} h\left(t_{0}, t_{1}\right)=-\frac{R_{0}\left(R_{0}+R_{1}\right)^{2}}{\left(t_{1}-t_{0}\right)^{3}}+o\left(\frac{1}{\left(t_{1}-t_{0}\right)^{3}}\right)
$$

as $\left(t_{1}-t_{0}\right) \rightarrow 0^{+}$. Hence, (iii) is proved.

Let $\Omega \subset \mathbb{R}^{2}$ be the strip defined in Proposition 4.3 and rewrite $h: \Omega \rightarrow \mathbb{R}$ as

$$
\begin{aligned}
h\left(t_{0}, t_{1}\right)= & \frac{R_{0}^{2}+R_{1}^{2}+2 \sqrt{R_{0}^{2} R_{1}^{2}-c^{2}\left(t_{1}-t_{0}\right)^{2}}}{2\left(t_{1}-t_{0}\right)} \\
& +c \arctan \left(\frac{c\left(t_{1}-t_{0}\right)}{\sqrt{R_{0}^{2} R_{1}^{2}-c^{2}\left(t_{1}-t_{0}\right)^{2}}}\right)
\end{aligned}
$$

where $R_{0}:=R\left(t_{0}\right), R_{1}:=R\left(t_{1}\right)$, and $c \in\left(0, \varepsilon \frac{\underline{R}^{2}}{\sigma}\right)$. As before let $\partial_{1}$ and $\partial_{2}$ denote the partial derivatives with respect to the first and second argument of a function, and let $\mathbb{T}$ denote the one-dimensional torus. 
Proposition 4.4. Define $\sigma_{*}:=\max _{t \in \mathbb{T}} \partial_{1} h(t, t+\sigma)$. The equations

$$
\left\{\begin{array}{l}
K_{0}=\partial_{1} h\left(t_{0}, t_{1}\right) \\
K_{1}=-\partial_{2} h\left(t_{0}, t_{1}\right)
\end{array}\right.
$$

define implicitly a $C^{k}$ embedding $P: \mathbb{T} \times\left(\sigma_{*},+\infty\right) \rightarrow \mathbb{T} \times \mathbb{R}, P\left(t_{0}, K_{0}\right)=$ $\left(t_{1}, K_{1}\right)$. Moreover, $P$ is twist in the sense that

$$
\frac{\partial t_{1}}{\partial K_{0}}<0
$$

and exact symplectic in the sense that there exists a $C^{k}$ function $V: \mathbb{T} \times \mathbb{R} \rightarrow \mathbb{R}$ such that

$$
K_{1} d t_{1}-K_{0} d t_{0}=d V\left(t_{0}, K_{0}\right) .
$$

Proof. Using that $\partial_{1} h\left(t_{0}, t_{1}\right) \rightarrow+\infty$ as $\left(t_{1}-t_{0}\right) \rightarrow 0^{+}$as can be shown from (4.6) and Proposition 4.3-(iii), we can apply the implicit function theorem to the first of (4.8) and get the $C^{k}$ function

$$
t_{1}=t_{1}\left(t_{0}, K_{0}\right)
$$

for $\left(t_{0}, K_{0}\right) \in \mathbb{T} \times\left(\sigma_{*},+\infty\right)$. Inserting (4.9) into the second of (4.8), we get the desired $C^{k}$ map $P$. To prove that it is injective, we note that if $P\left(t_{0}, K_{0}\right)=P\left(t_{0}^{\prime}, K_{0}^{\prime}\right)$ then, using once again Proposition 4.3-(iii), the second of (4.8) implies $t_{0}=t_{0}^{\prime}$ that, substituted in the first gives also $K_{0}=K_{0}^{\prime}$. By implicit differentiation of the first, we get the twist condition:

$$
\frac{\partial t_{1}}{\partial K_{0}}=\frac{1}{\partial_{12} h\left(t_{0}, t_{1}\right)}<0 .
$$

Finally, if we define $V\left(t_{0}, K_{0}\right)=-h\left(t_{0}, t_{1}\left(t_{0}, K_{0}\right)\right)$ we get

$$
\begin{aligned}
d V\left(t_{0}, K_{0}\right) & =\left(-h_{1}\left(t_{0}, t_{1}\right)-h_{2}\left(t_{0}, t_{1}\right) \frac{\partial t_{1}}{\partial t_{0}}\right) d t_{0}-h_{2}\left(t_{0}, t_{1}\right) \frac{\partial t_{1}}{\partial K_{0}} d K_{0} \\
& =-K_{0} d t_{0}+K_{1} \frac{\partial t_{1}}{\partial t_{0}} d t_{0}+K_{1} \frac{\partial t_{1}}{\partial K_{0}} d K_{0}=K_{1} d t_{1}-K_{0} d t_{0}
\end{aligned}
$$

Remark 4.5. It follows from the previous Proposition that a sequence $\left(t_{n}, K_{n}\right)$ is an orbit of the map $P$ if and only if, for every $n,\left(t_{n+1}-t_{n}\right) \in \Omega$ and

$$
\partial_{1} h\left(t_{n}, t_{n+1}\right)+\partial_{2} h\left(t_{n-1}, t_{n}\right)=0, \quad K_{n}=\partial_{1} h\left(t_{n}, t_{n+1}\right) .
$$

We finally note that the orbits of the diffeomorphism $P$ give rise to bouncing solutions of the billiard map in the breathing circle $\mathcal{D}_{t}$.

Proposition 4.6. Let $\left(t_{n}, K_{n}\right)$ be an orbit of $P$, let us set $R_{n}:=R\left(t_{n}\right)$ and define $\dot{r}\left(t_{n}^{+}\right)$as

$$
\dot{r}\left(t_{n}^{+}\right):=\dot{R}\left(t_{n}\right)-\sqrt{\dot{R}^{2}\left(t_{n}\right)-2 K_{n}-\frac{c^{2}}{R_{n}^{2}}},
$$

then $\left\{\left(t_{n}, \dot{r}\left(t_{n}^{+}\right)\right)\right\}$represents a bouncing solution in the sense that $\left\{t_{n}\right\}$ is the sequence of bouncing times, and $\left\{\dot{r}\left(t_{n}^{+}\right)\right\}$is the sequence of radial velocities 
right after the bounce at time $t_{n}$ with corresponding trajectories between two consecutive bounces being the solutions to system (3.1) found in Proposition 3.1 .

Proof. Using (3.16), we write

$$
K_{n}=-\frac{1}{2} A\left(t_{n}, t_{n+1}\right)-\dot{R}\left(t_{n}\right) \frac{R_{n}^{2}+\sqrt{R_{n}^{2} R_{n+1}^{2}-c^{2}\left(t_{n+1}-t_{n}\right)^{2}}}{R_{n}\left(t_{n+1}-t_{n}\right)} .
$$

Since

$$
A\left(t_{n}, t_{n+1}\right)-\frac{c^{2}}{R_{n}^{2}}=\left(\frac{R_{n}^{2}+\sqrt{R_{n}^{2} R_{n+1}^{2}-c^{2}\left(t_{n+1}-t_{n}\right)^{2}}}{R_{n}\left(t_{n+1}-t_{n}\right)}\right)^{2}
$$

we obtain that $\dot{r}\left(t_{n}^{+}\right)$defined in (4.10) can be written as

$$
\begin{aligned}
\dot{r}\left(t_{n}^{+}\right) & =\dot{R}\left(t_{n}\right)-\sqrt{\left(\dot{R}\left(t_{n}\right)+\frac{R_{n}^{2}+\sqrt{R_{n}^{2} R_{n+1}^{2}-c^{2}\left(t_{n+1}-t_{n}\right)^{2}}}{R_{n}\left(t_{n+1}-t_{n}\right)}\right)^{2}} \\
& =-\frac{R_{n}^{2}+\sqrt{R_{n}^{2} R_{n+1}^{2}-c^{2}\left(t_{n+1}-t_{n}\right)^{2}}}{R_{n}\left(t_{n+1}-t_{n}\right)}
\end{aligned}
$$

where we have used that

$$
\begin{aligned}
& \frac{R_{n}^{2}+\sqrt{R_{n}^{2} R_{n+1}^{2}-c^{2}\left(t_{n+1}-t_{n}\right)^{2}}}{R_{n}\left(t_{n+1}-t_{n}\right)} \\
& >2\|\dot{R}\| \frac{R_{n}^{2}+\sqrt{R_{n}^{2} R_{n+1}^{2}-c^{2}\left(t_{n+1}-t_{n}\right)^{2}}}{R_{n} \underline{R}} \geq 2\|\dot{R}\|>-\dot{R}\left(t_{n}\right)
\end{aligned}
$$

since $\left(t_{n+1}-t_{n}\right)<\sigma$. A straightforward computation shows that $\dot{r}\left(t_{n}^{+}\right)$is then equal to $\dot{r}\left(t_{n}^{+} ; t_{n}, t_{n+1}\right)$, the velocity of solution (3.15) to system (3.1) found in Proposition 3.1. In particular, (4.11) shows that $\dot{r}\left(t_{n}^{+}\right)$satisfies the first condition in (3.8).

Remark 4.7. The definition of $\dot{r}\left(t_{n}^{+}\right)$in Proposition 4.6 is inspired by (4.3). Notice that the two solutions of (4.3) are

$$
\dot{r}_{ \pm}:=\dot{R}\left(t_{n}\right) \pm \sqrt{\dot{R}^{2}\left(t_{n}\right)+2 \partial_{t_{n}} h\left(t_{n}, t_{n+1}\right)-\frac{c^{2}}{R_{n}^{2}}}
$$

which can be written as

$$
\dot{r}_{ \pm}:=\dot{R}\left(t_{n}\right) \pm\left(\dot{R}\left(t_{n}\right)+\frac{R_{n}^{2}+\sqrt{R_{n}^{2} R_{n+1}^{2}-c^{2}\left(t_{n+1}-t_{n}\right)^{2}}}{R_{n}\left(t_{n+1}-t_{n}\right)}\right) .
$$

Now, since $\left(t_{n+1}-t_{n}\right)<\sigma$,

$$
\dot{r}_{+}=2 \dot{R}\left(t_{n}\right)+\frac{R_{n}^{2}+\sqrt{R_{n}^{2} R_{n+1}^{2}-c^{2}\left(t_{n+1}-t_{n}\right)^{2}}}{R_{n}\left(t_{n+1}-t_{n}\right)}
$$




$$
\begin{aligned}
& >2 \dot{R}\left(t_{n}\right)+2\|\dot{R}\| \frac{R_{n}^{2}+\sqrt{R_{n}^{2} R_{n+1}^{2}-c^{2}\left(t_{n+1}-t_{n}\right)^{2}}}{R_{n} \underline{R}} \\
& \geq 2 \dot{R}\left(t_{n}\right)+2\|\dot{R}\| \geq \max \left\{0, \dot{R}\left(t_{n}\right)\right\} .
\end{aligned}
$$

Hence, $\dot{r}_{-}$is the only solution of (4.3) which may represent the radial velocity of a bouncing solution leaving the boundary. Moreover, $\dot{r}_{-}=-\dot{r}_{+}+2 \dot{R}\left(t_{n}\right)$; hence, $\dot{r}_{+}$and $\dot{r}_{-}$can be interpreted as the radial velocity before and after the bounce, respectively.

Remark 4.8. For completeness, we show that the map $P$ defined in Proposition 4.4 corresponds to the map $\mathcal{M}$ considered in [29] with a different choice of variables. The variables used in $[29]$ are $(t, I)$, and the map $\mathcal{M}:\left(t_{0}, I_{0}\right) \mapsto$ $\left(t_{1}, I_{1}\right)$ is implicitly given by

$$
\left\{\begin{array}{l}
I_{1}=-I_{0}-2 R_{1} \dot{R}\left(t_{1}\right)+\frac{c^{2}+I_{0}^{2}}{R_{0}^{2}}\left(t_{1}-t_{0}\right) \\
\left(t_{1}-t_{0}\right)\left(\frac{c^{2}+I_{0}^{2}}{R_{0}^{2}}\left(t_{1}-t_{0}\right)-2 I_{0}\right)=R_{1}^{2}-R_{0}^{2}
\end{array}\right.
$$

with $I_{n}=-R_{n} \dot{r}\left(t_{n}^{+}\right)$in our notations. The second equation is obtained by

$$
\frac{c^{2}+I_{0}^{2}}{R_{0}^{2}}=\frac{c^{2}}{R_{0}^{2}}+\dot{r}^{2}\left(t_{0}^{+}\right)=A\left(t_{0}, t_{1}\right)
$$

and using (3.16) and (4.11) for $\dot{r}\left(t_{0}^{+}\right)$. On the other hand, from Proposition 4.6 and (4.8)

$$
\dot{r}\left(t_{1}^{+}\right)=\dot{R}\left(t_{1}\right)-\sqrt{\dot{R}^{2}\left(t_{1}\right)-2 \partial_{2} h\left(t_{0}, t_{1}\right)-\frac{c^{2}}{R_{1}^{2}}}
$$

and, arguing as in the proof of Proposition 4.3, it holds

$$
\begin{aligned}
\partial_{2} h\left(t_{0}, t_{1}\right)+\frac{c^{2}}{2 R_{1}^{2}}=\dot{R}\left(t_{1}\right) & \frac{R_{1}^{2}+\sqrt{R_{0}^{2} R_{1}^{2}-c^{2}\left(t_{1}-t_{0}\right)^{2}}}{R_{1}\left(t_{1}-t_{0}\right)} \\
- & \frac{1}{2}\left(\frac{R_{1}^{2}+\sqrt{R_{0}^{2} R_{1}^{2}-c^{2}\left(t_{1}-t_{0}\right)^{2}}}{R_{1}\left(t_{1}-t_{0}\right)}\right)^{2} .
\end{aligned}
$$

Therefore, using

$$
\frac{R_{1}^{2}+\sqrt{R_{0}^{2} R_{1}^{2}-c^{2}\left(t_{1}-t_{0}\right)^{2}}}{R_{1}\left(t_{1}-t_{0}\right)}>\frac{2 R_{1}\|\dot{R}\|}{\underline{R}} \geq 2\|\dot{R}\|>\dot{R}\left(t_{1}\right)
$$

for $\left(t_{1}-t_{0}\right)<\sigma$, we have

$$
\begin{aligned}
\dot{r}\left(t_{1}^{+}\right) & =\dot{R}\left(t_{1}\right)-\left(\frac{R_{1}^{2}+\sqrt{R_{0}^{2} R_{1}^{2}-c^{2}\left(t_{1}-t_{0}\right)^{2}}}{R_{1}\left(t_{1}-t_{0}\right)}-\dot{R}\left(t_{1}\right)\right) \\
& =2 \dot{R}\left(t_{1}\right)-\frac{R_{1}^{2}+\sqrt{R_{0}^{2} R_{1}^{2}-c^{2}\left(t_{1}-t_{0}\right)^{2}}}{R_{1}\left(t_{1}-t_{0}\right)} .
\end{aligned}
$$

Using (4.12), we get

$$
I_{1}=-R_{1} \dot{r}\left(t_{1}^{+}\right)=-2 R_{1} \dot{R}\left(t_{1}\right)+\frac{R_{1}^{2}+\sqrt{R_{0}^{2} R_{1}^{2}-c^{2}\left(t_{1}-t_{0}\right)^{2}}}{t_{1}-t_{0}}
$$


and again by (4.11) for $\dot{r}\left(t_{0}^{+}\right)$, we conclude

$$
\begin{aligned}
I_{1}+I_{0} & =-2 R_{1} \dot{R}\left(t_{1}\right)+\frac{R_{0}^{2}+R_{1}^{2}+2 \sqrt{R_{0}^{2} R_{1}^{2}-c^{2}\left(t_{1}-t_{0}\right)^{2}}}{t_{1}-t_{0}} \\
& =-2 R_{1} \dot{R}\left(t_{1}\right)+\left(t_{1}-t_{0}\right) A\left(t_{0}, t_{1}\right)
\end{aligned}
$$

which is the first equation.

\section{Periodic and Quasi-Periodic Orbits}

In this section, we give the proof of Theorem 2.5. The proof is based on the Aubry-Mather theory, and the results we use are recalled in Appendix B. We begin with a preliminary result for the billiard map. Let $\varepsilon \in(0,1)$ be a fixed parameter as in Definition 2.2.

Proposition 5.1. Suppose that $\sigma>2$ and fix $c \in\left(0, \varepsilon \frac{\underline{R}^{2}}{\sigma}\right)$. Then, for every $1<\omega<\sigma-1$

- if $\omega=p / q \in \mathbb{Q}$, then there exists a minimal orbit $\left(t_{n}, K_{n}\right)_{n \in \mathbb{Z}}$ of angular momentum $c$ of the billiard map such that $\left(t_{n+q}, K_{n+q}\right)=\left(t_{n}+p, K_{n}\right)$;

- if $\omega \in \mathbb{R} \backslash \mathbb{Q}$, then there exists a minimal invariant set $M_{\omega}$ of rotation number $\omega$ made of orbits of angular momentum c. Moreover, $M_{\omega}$ is the graph of a Lipschitz function $u: \pi\left(M_{\omega}\right) \rightarrow \mathbb{R}$, and $M_{\omega}$ is either an invariant curve or a Cantor set.

Proof. Fix $\sigma>2$ and $c \in\left(0, \varepsilon \frac{\underline{R}^{2}}{\sigma}\right)$. The function $h$ defined in (4.5) is by Proposition 4.3 a generating function when restricted to the set $\Omega=\left\{\left(t_{0}, t_{1}\right) \in\right.$ $\left.\mathbb{R}^{2}: 0<t_{1}-t_{0}<\sigma\right\}$. Choose $\omega$ such that $1<\omega<\sigma-1$. Fix a positive number $\beta<\min \{\omega-1, \sigma-\omega-1\}$. By compactness, there exists $\delta$ such that

$$
h_{12} \leq \delta<0 \quad \text { on } \Omega_{\beta}=\left\{\left(t_{0}, t_{1}\right) \in \mathbb{R}^{2}: \beta \leq t_{1}-t_{0} \leq \sigma-\beta\right\} .
$$

Hence, we can apply Lemma B.3 and find a generating function $\tilde{h}$ that coincides with $h$ on $\Omega_{\beta}$ and satisfies the hypothesis of Theorem B.4. The function $\tilde{h}$ generates a diffeomorphism $\tilde{P}$ that coincide with the billiard map on some strip. Applying Theorem B.4, for every $\tilde{\omega} \in \mathbb{R}$ we find the periodic orbits and the invariant sets $M_{\tilde{\omega}}$ described in the same theorem. These sets are made of orbits $\left(t_{n}, K_{n}\right)_{n \in \mathbb{Z}}$ of the diffeomorphism $\tilde{P}$ and become orbits of the billiard map if

$$
\left(t_{n}, t_{n+1}\right) \in \Omega_{\beta} \quad \text { for every } n \in \mathbb{Z} .
$$

For $\tilde{\omega}=\omega$, by (B.2), we have that

$$
\left|t_{n+1}-t_{n}-\omega\right| \leq 1
$$

that implies, since $\omega>1$, that for every $n \in \mathbb{Z}$

$$
0<\omega-1 \leq t_{n+1}-t_{n} \leq \omega+1 .
$$

By the choice of $\beta$, for every $n \in \mathbb{Z}$,

$$
\beta<\omega-1 \leq t_{n+1}-t_{n} \leq \omega+1<\sigma-\beta
$$


that is $\left(t_{n}, t_{n+1}\right) \in \Omega_{\beta}$ for every $n \in \mathbb{Z}$.

Then Corollary B.5 immediately implies

Corollary 5.2. For each $1<\omega<\sigma-1$, there exist two functions $\phi, \eta: \mathbb{R} \rightarrow \mathbb{R}$ such that for every $\xi \in \mathbb{R}$

$$
\begin{aligned}
& \phi(\xi+1)=\phi(\xi)+1, \quad \eta(\xi+1)=\eta(\xi), \\
& S(\phi(\xi), \eta(\xi))=(\phi(\xi+\omega), \eta(\xi+\omega))
\end{aligned}
$$

where $\phi$ is monotone (strictly if $\omega \in \mathbb{R} \backslash \mathbb{Q}$ ) and $\eta$ is of bounded variation. Moreover, $\phi$ and $\eta$ have the same points of continuity, and if $\xi$ is a point of continuity then so are $\xi \pm \omega$ and $\xi \pm 1$.

Let us now come to the

Proof of Theorem 2.5. Fix $c \in\left(0, \varepsilon \frac{\underline{R}^{2}}{\sigma}\right)$. Consider $\omega \in \mathbb{R} \backslash \mathbb{Q}$ and the corresponding functions $\phi, \eta: \mathbb{R} \rightarrow \mathbb{R}$ given by Corollary 5.2 . Denote by

$$
x_{\xi}(t)=(r(t), \theta(t))_{\xi}
$$

the bouncing solution with angular momentum $c$ which satisfies

$$
r(\phi(\xi))=R(\phi(\xi)), \quad \dot{r}(\phi(\xi))=\dot{R}(\phi(\xi))-\sqrt{\dot{R}^{2}(\phi(\xi))-2 \eta(\xi)-\frac{c^{2}}{R_{\phi(\xi)}^{2}}} .
$$

Since the system is rotationally invariant, it is clear that to fix $c, r(\phi(\xi))$ and $\dot{r}(\phi(\xi))$ are sufficient to uniquely determine the bouncing solution up to rotations. The value of $\theta(\phi(\xi))$ can be chosen freely. We claim that the family $\left\{x_{\xi}(t)\right\}_{\xi \in \mathbb{R}}$ satisfies the conditions in Definition 2.4.

By the periodicity of $\phi, \eta$,

$$
(r(t), \theta(t))_{\xi+1}=(r(t-1), \theta(t-1))_{\xi}
$$

In addition, using (5.2) and Proposition 4.6 we have

$$
(r(t), \theta(t))_{\xi+\omega}=(r(t), \theta(t))_{\xi} .
$$

Thus, we have proved that for every $\xi \in \mathbb{R}$

$$
\begin{aligned}
& x_{\xi+1}(t)=x_{\xi}(t-1), \\
& x_{\xi}(t)=x_{\xi+\omega}(t) .
\end{aligned}
$$

Consider now the function

$$
\Phi_{\xi}(a, b)=x_{\xi-\omega b+a}(a) .
$$

Using (5.3) and (5.4), it is not difficult to show that

$$
\begin{aligned}
& \Phi_{\xi}(a+1, b)=x_{\xi-\omega b+a+1}(a+1)=\Phi_{\xi}(a, b), \\
& \Phi_{\xi}(a, b+1)=x_{\xi-\omega b+a-\omega}(a)=\Phi_{\xi}(a, b)
\end{aligned}
$$

and $\Phi_{\xi}(t, t / \omega)=x_{\xi}(t)$. So that the solutions satisfy condition (i) in Definition 2.4, since the regularity follows from (5.5) and Corollary 5.2. Finally, condition (ii) follows from the definition of the rotation number of a minimal orbit. 


\section{Chaotic Motion}

In this section, we prove Theorem 2.7, showing the existence of chaotic motion for the billiard map inside the breathing circle $\mathcal{D}_{t}$ with $R(t) \in \widetilde{\mathcal{R}}$. In particular, we are going to prove that there exists $c_{0}>0$ such that for every $c \in\left(0, c_{0}\right)$ there exist many probability measures with positive metric entropy, which are invariant for the map $P$ defined in Proposition 4.4. As in the previous section, we will refer to results from Appendix B.

The idea of the proof is the following. First we extend the map $P$ to the whole cylinder as in the proof of Proposition 5.1. The key point is then to prove that there exists an open interval $\mathcal{I} \subset \mathbb{R}$ such that for sufficiently small values of $c$, the extended map has no invariant curve with rotation number $\omega \in \mathcal{I}$. Hence, for irrational $\omega \in \mathcal{I}$ the Mather sets $M_{\omega}$ of Theorem B.4 are Cantor sets. Then, Theorem B.6 guarantees the existence of invariant probability measures with positive metric entropy for the extended map. The final step is to show that the extension has been made in such a way that these invariant measures are supported in the zone of the cylinder where the extended map coincides with $P$.

Let us first state and prove a series of technical lemmas. Let $R \in \widetilde{\mathcal{R}}$ and recall by Definition 2.2 that $\sigma>4$. Moreover, in this section we use the notations $\dot{R}_{\bar{t}}$ and $\ddot{R}_{\bar{t}}$ for $\dot{R}(\bar{t})$ and $\ddot{R}(\bar{t})$, respectively. Let us consider the set

$\Xi_{R}:=\left\{\omega \in(3, \sigma-1): \frac{2 \bar{R}^{2}}{\sigma^{2}}<\frac{2 \underline{R}^{2}}{(\omega+1)^{2}}-\|\dot{R}\| \frac{2 \bar{R}}{\omega+1}<\frac{2 \bar{R}^{2}}{(\omega-1)^{2}}+\|\dot{R}\| \frac{2 \bar{R}}{\omega-1}<-\ddot{R}_{\bar{t}} \underline{R}\right\}$.

Lemma 6.1. If $R(t) \in \widetilde{\mathcal{R}}$, the set $\Xi_{R}$ is not empty and contains an open interval $\mathcal{I}$.

Proof. Let us first note that

$$
\frac{2 \underline{R}^{2}}{\frac{2 \bar{R}^{2}}{\sigma^{2}}+\|\dot{R}\| \bar{R}}<\frac{\underline{R}^{2}}{\bar{R}^{2}} \sigma^{2}<\sigma^{2}
$$

from which, by conditions (i) and (ii) of Definition 2.2, there exist $3<\omega^{-}<$ $\omega^{+}<\sigma-1$ such that every $\omega \in\left(\omega^{-}, \omega^{+}\right)$satisfies

$$
1+\sqrt{\frac{2 \bar{R}^{2}}{-\ddot{R}_{\bar{t}} \underline{R}-\|\dot{R}\| \bar{R}}}<\omega<-1+\sqrt{\frac{2 \underline{R}^{2}}{\frac{2 \bar{R}^{2}}{\sigma^{2}}+\|\dot{R}\| \bar{R}}}
$$

or, equivalently,

$$
(\omega-1)^{2}>\frac{2 \bar{R}^{2}}{-\ddot{R}_{\bar{t}} \underline{R}-\|\dot{R}\| \bar{R}} \quad \text { and } \quad(\omega+1)^{2}<\frac{2 \underline{R}^{2}}{\frac{2 \bar{R}^{2}}{\sigma^{2}}+\|\dot{R}\| \bar{R}} .
$$

Since $\omega>3$, using the first of $(6.1)$

$$
\frac{2 \bar{R}^{2}}{(\omega-1)^{2}}+\|\dot{R}\| \frac{2 \bar{R}}{\omega-1}<\frac{2 \bar{R}^{2}}{(\omega-1)^{2}}+\|\dot{R}\| \bar{R}<-\ddot{R}_{\bar{t}} \underline{R}
$$


that proves the third inequality in the definition of the set $\Xi_{R}$. Analogously, since $\omega>1$, using the second of (6.1)

$$
\frac{2 \underline{R}^{2}}{(\omega+1)^{2}}-\|\dot{R}\| \frac{2 \bar{R}}{\omega+1}>\frac{2 \underline{R}^{2}}{(\omega+1)^{2}}-\|\dot{R}\| \bar{R}>\frac{2 \bar{R}^{2}}{\sigma^{2}} .
$$

that proves the first inequality in the definition of the set $\Xi_{R}$. The second inequality can be easily proved.

Lemma 6.2. Let $c \in\left(0, \varepsilon \frac{R^{2}}{\sigma}\right)$ and $\mathcal{I}=\left(\omega^{-}, \omega^{+}\right)$be the interval defined in Lemma 6.1. Let $\Gamma=\{(t, \gamma(t)): t \in \mathbb{T}\}$ be an invariant curve of the billiard map with rotation number $\omega \in \mathcal{I}$. Then

$$
\mathcal{K}^{-}(\omega)+o(c) \leq \gamma(t) \leq \mathcal{K}^{+}(\omega)+o(c),
$$

where

$$
\mathcal{K}^{-}(\omega)=\frac{2 \underline{R}^{2}}{(\omega+1)^{2}}-\|\dot{R}\| \frac{2 \bar{R}}{\omega+1}, \quad \mathcal{K}^{+}(\omega)=\frac{2 \bar{R}^{2}}{(\omega-1)^{2}}+\|\dot{R}\| \frac{2 \bar{R}}{\omega-1}
$$

and $o(c)$ represents a function depending on $R, \omega, c, t_{0}, t_{1}$ that tends to zero uniformly for $\omega \in \mathcal{I}$ as $c \rightarrow 0^{+}$.

Proof. Let $\left(t_{n}, K_{n}\right)$ be an orbit of the billiard map with rotation number $\omega$ on the invariant curve $\Gamma$. From (4.8) and (4.6), a direct computation gives for the point $\left(t_{0}, K_{0}\right)$ of the orbit

$$
K_{0}=\frac{1}{2}\left(\frac{R_{0}+R_{1}}{t_{1}-t_{0}}\right)^{2}+\dot{R}\left(t_{0}\right)\left(\frac{R_{0}+R_{1}}{t_{1}-t_{0}}\right)-c^{2} f\left(t_{1}, t_{0}, c\right)
$$

where

$$
f\left(t_{1}, t_{0}, c\right)=\frac{1}{2 R_{0}}+\frac{R_{0}+\dot{R}\left(t_{0}\right)\left(t_{1}-t_{0}\right)}{R_{0}^{2} R_{1}+R_{0} \sqrt{R_{0}^{2} R_{1}^{2}-c^{2}\left(t_{1}-t_{0}\right)^{2}}}
$$

can be bounded by a constant depending on $R$ and $c$ but not on $\omega$. Actually, from (B.2) and the fact that $\omega \in \mathcal{I}$,

$$
2<\omega^{-}-1<\omega-1<t_{1}\left(t_{0}, K_{0}, c\right)-t_{0}<\omega+1<\omega^{+}+1
$$

so that $\left|t_{1}\left(t_{0}, K_{0}, c\right)-t_{0}\right|$ is uniformly bounded on every invariant curve with rotation number $\omega \in \mathcal{I}$ for $c$ fixed. Solving (6.2) for $\left(t_{1}-t_{0}\right)$, we get

$$
t_{1}-t_{0}=\frac{R_{0}+R_{1}}{\sqrt{\dot{R}^{2}\left(t_{0}\right)+2\left(K_{0}+c^{2} f\left(t_{1}, t_{0}, c\right)\right)}-\dot{R}\left(t_{0}\right)}
$$

that used in (6.3) gives

$$
\mathcal{K}^{-}(\omega) \leq K_{0}+c^{2} f\left(t_{1}, t_{0}, c\right) \leq \mathcal{K}^{+}(\omega) .
$$

Since this argument applies to all points of $\Gamma$, the proof is finished. 
Lemma 6.3. Let $\mathcal{I}=\left(\omega^{-}, \omega^{+}\right)$be the interval defined in Lemma 6.1. Suppose that there exists an invariant curve $\Gamma$ of the billiard map with rotation number $\omega \in \mathcal{I}$. Consider a point $(\bar{t}, \bar{K}) \in \Gamma$ such that

$$
\dot{R}_{\bar{t}}=0
$$

Let $t_{1}=t_{1}(\bar{t}, K, c)$ and $t_{-1}=t_{-1}(\bar{t}, K, c)$, and consider the function

$$
a_{c}(\bar{t}, K):=a\left(t_{-1}(\bar{t}, K, c), \bar{t}, t_{1}(\bar{t}, K, c)\right)
$$

with the notation given in Proposition B.7. Then

$$
a_{c}(\bar{t}, K)=2 \sqrt{2 K}\left(\ddot{R}_{\bar{t}}+K\left(\frac{1}{R_{0}+R_{\bar{t}}}+\frac{1}{R_{2}+R_{\bar{t}}}\right)\right)+o(c),
$$

where o $(c)$ represents a function depending on $R, \omega, c, t_{0}, t_{1}$ that tends to zero uniformly for $\omega \in \mathcal{I}$ as $c \rightarrow 0$.

Proof. Let $h(t, s)$ be the function defined in (4.5) on the the strip $\Omega=\{(t, s) \in$ $\left.\mathbb{R}^{2}: 0<t-s<\sigma\right\}$, with $\varepsilon, \sigma$ and $c$ given as in Proposition 4.3. Computations show that

$$
\begin{aligned}
\partial_{11} h(t, s)= & \ddot{R}(t) \frac{R_{t}^{2}+\sqrt{R_{t}^{2} R_{s}^{2}-c^{2}(s-t)^{2}}}{R_{t}(s-t)}+\frac{\dot{R}^{2}(t)}{s-t}\left(1+\frac{c^{2}(s-t)^{2}}{R_{t}^{2} \sqrt{R_{t}^{2} R_{s}^{2}-c^{2}(s-t)^{2}}}\right) \\
& +2 \frac{R_{t} \dot{R}(t)}{(s-t)^{2}}\left(1+\frac{R_{t}^{2} R_{s}^{2}}{R_{t}^{2} \sqrt{R_{t}^{2} R_{s}^{2}-c^{2}(s-t)^{2}}}\right) \\
& +\frac{A(t, s)}{s-t}+\frac{c^{2}(s-t)^{2}}{(s-t)^{3} \sqrt{R_{t}^{2} R_{s}^{2}-c^{2}(s-t)^{2}}} \\
\partial_{22} h(t, s)= & \ddot{R}(s) \frac{R_{s}^{2}+\sqrt{R_{t}^{2} R_{s}^{2}-c^{2}(s-t)^{2}}}{R_{s}(s-t)} \\
& +\frac{\dot{R}^{2}(s)}{s-t}\left(1+\frac{c^{2}(s-t)^{2}}{R_{s}^{2} \sqrt{R_{t}^{2} R_{s}^{2}-c^{2}(s-t)^{2}}}\right) \\
& -2 \frac{R_{s} \dot{R}(s)}{(s-t)^{2}}\left(1+\frac{R_{t}^{2} R_{s}^{2}}{R_{s}^{2} \sqrt{R_{t}^{2} R_{s}^{2}-c^{2}(s-t)^{2}}}\right) \\
& +\frac{A(t, s)}{s-t}+\frac{c^{2}(s-t)^{2}}{(s-t)^{3} \sqrt{R_{t}^{2} R_{s}^{2}-c^{2}(s-t)^{2}}}
\end{aligned}
$$

where $R_{t}:=R(t)$ and $R_{s}:=R(s)$, and we recall from (3.16) that

$$
A(t, s)=\frac{R_{t}^{2}+R_{s}^{2}+2 \sqrt{R_{t}^{2} R_{s}^{2}-c^{2}(s-t)^{2}}}{(s-t)^{2}} .
$$

Consider the function 


$$
(t, K, c) \mapsto a_{c}(t, K):=\partial_{11} h\left(t, t_{1}\right)+\partial_{22} h\left(t_{-1}, t\right)
$$

then

$$
\begin{aligned}
a_{c}(t, K)= & 2 \ddot{R}(t)\left(\dot{R}(t)+\frac{R_{t}^{2}+\sqrt{R_{t}^{2} R_{1}^{2}-c^{2}\left(t_{1}-t\right)^{2}}}{R_{t}\left(t_{1}-t\right)}\right) \\
& +\frac{\dot{R}^{2}(t)}{t-t_{-1}}\left(1+\frac{c^{2}\left(t-t_{-1}\right)^{2}}{R_{t}^{2} \sqrt{R_{t}^{2} R_{-1}^{2}-c^{2}\left(t-t_{-1}\right)^{2}}}\right) \\
& +\frac{\dot{R}^{2}(t)}{t_{1}-t}\left(1+\frac{c^{2}\left(t_{1}-t\right)^{2}}{R_{t}^{2} \sqrt{R_{t}^{2} R_{1}^{2}-c^{2}\left(t_{1}-t\right)^{2}}}\right) \\
& +\frac{A\left(t_{-1}, t\right)}{t-t_{-1}}+\frac{\left(t-c_{-1}\right)^{3} \sqrt{R_{t}^{2} R_{-1}^{2}-c^{2}\left(t-t_{-1}\right)^{2}}}{\left(t, t_{1}\right)} \\
& +\frac{c^{2}\left(t_{1}-t\right)^{2}}{t_{1}-t}+\frac{R_{t}^{2} R_{1}^{2}}{\left(t_{1}-t\right)^{3} \sqrt{R_{t}^{2} R_{1}^{2}-c^{2}\left(t_{1}-t\right)^{2}}} \\
& +2 \frac{R_{t} \dot{R}(t)}{\left(t_{1}-t\right)^{2}}\left(1+\frac{R_{t}^{2}}{R_{t}^{2} \sqrt{R_{t}^{2} R_{1}^{2}-c^{2}\left(t_{1}-t\right)^{2}}}\right) \\
& -2 \frac{R_{t}^{2} R_{-1}^{2}}{(t-t)}\left(1+\frac{R_{-1}^{2} \sqrt{R_{t}^{2} R_{-1}^{2}-c^{2}\left(t-t_{-1}\right)^{2}}}{(1}\right)
\end{aligned}
$$

where we have used that

$$
\begin{aligned}
& \frac{R_{t}^{2}+\sqrt{R_{t}^{2} R_{-1}^{2}-c^{2}\left(t-t_{-1}\right)^{2}}}{R_{t}\left(t-t_{-1}\right)}=\dot{r}\left(t^{-} ; t_{-1}, t\right) \\
& =-\dot{r}\left(t^{+} ; t, t_{1}\right)+2 \dot{R}(t)=\frac{R_{t}^{2}+\sqrt{R_{t}^{2} R_{1}^{2}-c^{2}\left(t_{-1}-t\right)^{2}}}{R_{t}\left(t_{-1}-t\right)}+2 \dot{R}(t) .
\end{aligned}
$$

As in Lemma 6.2 , since $t_{1}(t, K, c)-t$ and $t-t_{-1}(t, K, c)$ are uniformly bounded for $c$ fixed, we can write

$$
\left\{\begin{array}{l}
K=\frac{1}{2}\left(\frac{R_{t}+R_{1}}{t_{1}-t}\right)^{2}+\dot{R}_{t} \frac{R_{t}+R_{1}}{t_{1}-t}+o(c) \\
K_{1}=\frac{1}{2}\left(\frac{R_{t}+R_{1}}{t_{1}-t}\right)^{2}-\dot{R}_{1} \frac{R_{t}+R_{1}}{t_{1}-t}+o(c)
\end{array}\right.
$$

from which

$$
\left\{\begin{array}{l}
t_{1}-t=\frac{R_{t}+R_{1}}{\sqrt{\dot{R}_{t}^{2}+2 K}-\dot{R}_{t}}+o(c) \\
t_{0}-t_{-1}=\frac{R_{-1}+R_{t}}{\sqrt{\dot{R}_{t}^{2}+2 K}+\dot{R}_{t}}+o(c) .
\end{array}\right.
$$

Using these formulas in the expression of $a_{c}(t, K)$ for $t=\bar{t}$, since $\dot{R}_{\bar{t}}=0$ we get from standard computations

$$
a_{c}(\bar{t}, K)=2 \sqrt{2 K}\left(\ddot{R}_{\bar{t}}+K\left(\frac{1}{R_{0}+R_{\bar{t}}}+\frac{1}{R_{2}+R_{\bar{t}}}\right)\right)+o(c)
$$


where $o(c)$ is as in Lemma 6.2.

Lemma 6.4. If $\dot{R}_{\bar{t}}=0$ and $\ddot{R}_{\bar{t}}<-\frac{2 \bar{R}^{2}}{\sigma^{2} \underline{R}}$, then

$$
\alpha(\bar{t}, K):=2 \sqrt{2 K}\left(\ddot{R}_{\bar{t}}+K\left(\frac{1}{R_{0}+R_{\bar{t}}}+\frac{1}{R_{2}+R_{\bar{t}}}\right)\right)<0
$$

for every $K \in\left(\frac{2 \bar{R}^{2}}{\sigma^{2}},-\ddot{R}_{\bar{t}} \underline{R}\right)$.

Proof. First note that

$$
\alpha(\bar{t}, K)=2 \sqrt{2 K}\left(\ddot{R}_{\bar{t}}+K\left(\frac{1}{R_{0}+R_{\bar{t}}}+\frac{1}{R_{2}+R_{\bar{t}}}\right)\right)<2 \sqrt{2 K}\left(\ddot{R}_{\bar{t}}+\frac{K}{\underline{R}}\right),
$$

from which we get $\alpha(\bar{t}, K)<0$ for every $K \in\left(0,-\ddot{R}_{\bar{t}} \underline{R}\right)$. Moreover, by the hypothesis on $\ddot{R}_{\bar{t}}$ it holds $0<\frac{2 \bar{R}^{2}}{\sigma^{2}}<-\ddot{R}_{\bar{t}} \underline{R}$.

We are now ready to extend the map $P$ to the cylinder $\mathbb{T} \times \mathbb{R}$. Fix $\sigma>4$. As in the proof of Proposition 5.1, for every $c \in\left(0, \varepsilon \frac{\underline{R}^{2}}{\sigma}\right)$, the function $h$ defined in (4.5) is, by Proposition 4.3, a generating function when restricted to the set $\Omega=\left\{\left(t_{0}, t_{1}\right) \in \mathbb{R}^{2}: 0<t_{1}-t_{0}<\sigma\right\}$. By Lemma 6.1, we can fix $\omega \in \mathcal{I}=\left(\omega^{-}, \omega^{+}\right) \subset \Xi_{R}$. Fix a positive number $\beta<\min \left\{\omega^{-}-1, \sigma-\omega^{+}-1\right\}$ and consider the set

$$
\Omega_{\beta}=\left\{\left(t_{0}, t_{1}\right) \in \mathbb{R}^{2}: \beta \leq t_{1}-t_{0} \leq \sigma-\beta\right\} .
$$

Hence, we can apply Lemma B.3 and find a generating function $\tilde{h}$ that coincides with $h$ on $\Omega_{\beta}$ and satisfies the assumptions of Theorem B.4. The function $\tilde{h}$ generates a diffeomorphism $\tilde{P}$ that coincides with the billiard map on some strip.

The following result is crucial for the proof of Theorem 2.7.

Proposition 6.5. Suppose that $R(t) \in \widetilde{\mathcal{R}}$. Then, there exists $c_{0}$ such that for every $c \in\left(0, c_{0}\right)$ and $\omega \in \mathcal{I} \subset \Xi_{R}$ the extended map $\tilde{P}$ does not admit any invariant curve with rotation number $\omega$.

Proof. Suppose by contradiction that for $c \rightarrow 0$, the map $\tilde{P}$ has an invariant curve $\Gamma$ with rotation number $\omega \in \mathcal{I}$. By (B.2), for every orbit $\left\{\left(t_{n}, K_{n}\right)\right\}$ on $\Gamma$ we have that

$$
\left|t_{n+1}-t_{n}-\omega\right| \leq 1
$$

that implies, since $\omega>\omega^{-}>3$, that for every $n \in \mathbb{Z}$

$$
0<\omega-1 \leq t_{n+1}-t_{n} \leq \omega+1 .
$$

By the choice of $\beta$, for every $n \in \mathbb{Z}$,

$$
\beta<\omega^{-}-1<\omega-1 \leq t_{n+1}-t_{n} \leq \omega+1<\omega^{+}+1<\sigma-\beta
$$

that is $\left(t_{n}, t_{n+1}\right) \in \Omega_{\beta}$ for every $n \in \mathbb{Z}$. Hence the dynamics on the invariant curve are given by the billiard map P. By condition (iii) in Definition 2.2, there exists a point $(\bar{t}, \bar{K}) \in \Gamma$ such that

$$
\dot{R}_{\bar{t}}=0 \quad \text { and } \quad \ddot{R}_{\bar{t}}<-\frac{2 \bar{R}^{2}}{\sigma^{2} \underline{R}} .
$$


By Proposition B.7 and Lemma 6.3,

$$
\begin{aligned}
a_{c}(\bar{t}, \bar{K}) & =\alpha(\bar{t}, \bar{K})+o(c) \\
& =2 \sqrt{2 \bar{K}}\left(\ddot{R}_{\bar{t}}+\bar{K}\left(\frac{1}{R_{0}+R_{\bar{t}}}+\frac{1}{R_{2}+R_{\bar{t}}}\right)\right)+o(c)>0 .
\end{aligned}
$$

However, from Lemma 6.4 we have $\alpha(\bar{t}, K)<0$ for every $K \in\left(\frac{2 \bar{R}^{2}}{\sigma^{2}},-\ddot{R}_{\bar{t}} \underline{R}\right)$ then, if

$$
\bar{K} \in\left(\frac{2 \bar{R}^{2}}{\sigma^{2}},-\ddot{R}_{\bar{t}} \underline{R}\right)
$$

for $c$ sufficiently small, we obtain a contradiction and the proposition is proved. In fact, applying Lemmas 6.1 and 6.2 we find

$$
\bar{K} \leq \frac{2 \bar{R}^{2}}{(\omega-1)^{2}}+\|\dot{R}\| \frac{2 \bar{R}}{\omega-1}+o(c)<-\ddot{R}_{\bar{t}} \underline{R}+o(c)
$$

and

$$
\bar{K} \geq \frac{2 \underline{R}^{2}}{(\omega+1)^{2}}-\|\dot{R}\| \frac{2 \bar{R}}{\omega+1}+o(c)>\frac{2 \bar{R}^{2}}{\sigma^{2}}+o(c),
$$

where $o(c)$ represents a function that tends to zero for $c \rightarrow 0$ uniformly for $\omega \in \mathcal{I}$, and we are done.

Proof of Theorem 2.7. Consider the extended map $\tilde{P}$ for $c<c_{0}$, where $c_{0}$ is given as in Proposition 6.5. For every irrational $\omega \in \mathcal{I} \subset \Xi_{R}$, the Mather set $M_{\omega}$ is a Cantor set and there are no invariant curves with rotation number $\omega$. Hence, Theorem B.6 gives, for every irrational $\omega \in \mathcal{I}$, the existence of a $\tilde{P}$-invariant measure $\mu_{\omega}$ with positive metric entropy arbitrarily close, in the sense specified in Theorem B.6, to the Mather set $M_{\omega}$. By the choice of the extension, as shown in Proposition 6.5, the Mather sets $M_{\omega}$ are contained in the zone of the cylinder where $\tilde{P}=P$. Hence, there exist measures $\mu_{\omega}$ which are $P$-invariant.

\section{Acknowledgements}

This research was partially supported by the PRIN Grant 2017S35EHN of the Ministry of Education, University and Research (MIUR), Italy. It is also part of the authors' activity within the DinAmicI community (www.dinamici. org) and the Gruppo Nazionale di Fisica Matematica, INdAM, Italy. We are also grateful to the referee whose suggestions significantly improved the final version of the paper.

Funding Open access funding provided by Universitá di Pisa within the CRUICARE Agreement. 
Open Access. This article is licensed under a Creative Commons Attribution 4.0 International License, which permits use, sharing, adaptation, distribution and reproduction in any medium or format, as long as you give appropriate credit to the original author(s) and the source, provide a link to the Creative Commons licence, and indicate if changes were made. The images or other third party material in this article are included in the article's Creative Commons licence, unless indicated otherwise in a credit line to the material. If material is not included in the article's Creative Commons licence and your intended use is not permitted by statutory regulation or exceeds the permitted use, you will need to obtain permission directly from the copyright holder. To view a copy of this licence, visit http://creativecommons. org/licenses/by/4.0/.

Publisher's Note Springer Nature remains neutral with regard to jurisdictional claims in published maps and institutional affiliations.

\section{A. The Classes $\mathcal{R}$ and $\widetilde{\mathcal{R}}$}

Proposition A.1. For a fixed $\varepsilon \in(0,1)$, let $\alpha:=\sqrt{1+\sqrt{1-\varepsilon^{2}}}$ and

$$
\bar{k}:=\frac{\alpha^{2}+\sqrt{2 \alpha^{4}-1}}{\alpha^{2}-1} .
$$

For every integer $k>\bar{k}$ and $\delta>0$ such that

$$
\frac{1}{4 \pi^{2}\left(k^{2}+1\right)}<\delta<\frac{1}{2 \pi(k+1)}
$$

there exists $M>0$ such that the function

$$
R_{k, \delta, M}(t):=M+\delta \sin (2 \pi k t)+\delta \sin (2 \pi t)
$$

belongs to the class $\widetilde{\mathcal{R}}$.

Moreover, if $\varepsilon<\sqrt{1-\frac{1}{(\pi-1)^{2}}}$, then there exist $\delta, M$ such that

$$
R_{\delta, M}(t):=M+\delta \sin (2 \pi t)
$$

is in $\widetilde{\mathcal{R}}$.

Remark A.2. Notice that for $\varepsilon=1$ the class $\widetilde{\mathcal{R}}$ is empty. From condition (iii) and the definition of $\sigma$,

$$
\ddot{R}(\bar{t})<-\frac{2 \bar{R}^{2}}{\sigma^{2} \underline{R}} \leq-\frac{\bar{R}^{2}}{2 \underline{R}^{3}}\left\|\frac{d^{2}}{d t^{2}} R^{2}\right\| .
$$

But

$$
\left\|\frac{d^{2}}{d t^{2}} R^{2}\right\| \geq\left|\frac{d^{2}}{d t^{2}} R^{2}\right|_{t=\bar{t}}|=2 R(\bar{t})| \ddot{R}(\bar{t})|\geq 2 \underline{R}| \ddot{R}(\bar{t}) \mid,
$$

so that

$$
\ddot{R}(\bar{t})<-\frac{\bar{R}^{2}}{\underline{R}^{2}}|\ddot{R}(\bar{t})|
$$

that is impossible. 
Proof of Proposition A.1. It is clear that for every $k \in \mathbb{N}, \delta>0$, the function $R(t)$ is $C^{2}, 1$-periodic and positive if $M>2 \delta$. It is easily seen that

$$
\underline{R}=M-2 \delta, \quad \bar{R}=M+2 \delta, \quad\|\dot{R}\|=2 \pi \delta(k+1)
$$

and

$$
\left\|\frac{\mathrm{d}^{2}}{\mathrm{~d} t^{2}} R^{2}\right\| \leq 8 \pi^{2} \delta\left[\left(k^{2}+1\right)(M+3 \delta)+2 k \delta\right] .
$$

Moreover, choosing $\bar{t}=\pi / 2$,

$$
\dot{R}(\bar{t})=0, \quad-\ddot{R}(\bar{t})=4 \pi^{2} \delta\left(k^{2}+1\right) .
$$

Finally it is immediate from the hypothesis that $\delta<1$.

Let us start with the computation of $\sigma$. Using (A.1), we have that

$$
\sigma=(M-2 \delta) \min \left\{\frac{1}{4 \pi \delta(k+1)}, \frac{2 \alpha}{\sqrt{\left\|\frac{d^{2}}{d t^{2}} R^{2}\right\|}}\right\}
$$

We note that $\left\|\frac{d^{2}}{d t^{2}} R^{2}\right\| \geq 2 \underline{R}(-\ddot{R}(\bar{t}))=8 \pi^{2} \delta(M-2 \delta)\left(k^{2}+1\right)$. If

$$
M>\max \left\{34 \delta, 2 \delta+216 \pi^{2}(k+1)^{2}\right\}
$$

using the fact that $\alpha \in(1, \sqrt{2})$ one can show that

$$
\begin{aligned}
4 \pi \delta(k+1) & <\frac{\sqrt{8 \pi^{2} \delta(M-2 \delta)\left(k^{2}+1\right)}}{4}<\frac{\sqrt{8 \pi^{2} \delta(M-2 \delta)\left(k^{2}+1\right)}}{2 \alpha} \\
& =\frac{\sqrt{2 \underline{R}(-\ddot{R}(\bar{t}))}}{2 \alpha}<\frac{\sqrt{\left\|\frac{d^{2}}{d t^{2}} R^{2}\right\|}}{2 \alpha}
\end{aligned}
$$

from which using (A.2) and (A.3)

$$
\sigma=\frac{2(M-2 \delta) \alpha}{\sqrt{\left\|\frac{d^{2}}{d t^{2}} R^{2}\right\|}}>\frac{2(M-2 \delta) \alpha}{\sqrt{8 \pi^{2} \delta\left[\left(k^{2}+1\right)(M+3 \delta)+2 k \delta\right]}}>4 .
$$

This gives condition $(i)$.

To prove that condition ( $i i i)$ holds we note that if

$$
M>2 \delta \frac{4 \pi^{2} \delta\left(k^{2}+1\right)+1}{4 \pi^{2} \delta\left(k^{2}+1\right)-1},
$$

using that by hypothesis $4 \pi^{2} \delta\left(k^{2}+1\right)-1>0$, we get

$$
-\ddot{R}(\bar{t})=4 \pi^{2} \delta\left(k^{2}+1\right)>\frac{M+2 \delta}{M-2 \delta}>\frac{2(M+2 \delta)}{16(M-2 \delta)}>\frac{2 \bar{R}}{\sigma^{2} \underline{R}}
$$

since $\sigma>4$.

Let us now prove condition $(i i)$. By (A.5) and the hypothesis $2 \pi \delta(k+1)<$ 1, it holds

$$
\begin{aligned}
& -\ddot{R}(\bar{t}) \underline{R}-\|\dot{R}\| \bar{R}=4 \pi^{2} \delta\left(k^{2}+1\right)(M-2 \delta) \\
& -2 \pi \delta(k+1)(M+2 \delta)>(M+2 \delta)(1-2 \pi \delta(k+1))>0 .
\end{aligned}
$$


Since by (A.3)

$$
M>8 \pi^{2} \delta\left(k^{2}+1\right)
$$

then

$$
\begin{aligned}
2 & <\sqrt{\frac{M}{2 \pi^{2} \delta\left(k^{2}+1\right)}}<\sqrt{\frac{2(M+2 \delta)^{2}}{4 \pi^{2} \delta\left(k^{2}+1\right)(M-2 \delta)-2 \pi \delta(k+1)(M+2 \delta)}} \\
& =\sqrt{\frac{2 \bar{R}^{2}}{-\ddot{R}(\bar{t}) \underline{R}-\|\dot{R}\| \bar{R}}}
\end{aligned}
$$

that gives the first inequality in $(i i)$. To prove the second inequality, we note that the first inequality in (A.4) gives

$$
\sqrt{\frac{2 \underline{R}^{2}}{\frac{2 \bar{R}^{2}}{\sigma^{2}}+\|\dot{R}\| \bar{R}}}>\sqrt{\frac{\alpha^{2}(M-2 \delta)^{4}}{\pi \delta\left[2 \pi(M+2 \delta)^{2}\left(\left(k^{2}+1\right)(M+3 \delta)+2 k \delta\right)+\alpha^{2}(k+1)(M+2 \delta)(M-2 \delta)^{2}\right]}}
$$

so that we are done if

$$
\begin{aligned}
1 & +\sqrt{\frac{2(M+2 \delta)^{2}}{4 \pi^{2} \delta\left(k^{2}+1\right)(M-2 \delta)-2 \pi \delta(k+1)(M+2 \delta)}}< \\
& -1+\sqrt{\frac{\alpha^{2}(M-2 \delta)^{4}}{\pi \delta\left[2 \pi(M+2 \delta)^{2}\left(\left(k^{2}+1\right)(M+3 \delta)+2 k \delta\right)+\alpha^{2}(k+1)(M+2 \delta)(M-2 \delta)^{2}\right]}}
\end{aligned}
$$

Looking at the asymptotic behaviour as $M \rightarrow+\infty$ of the left- and right-hand side of the previous inequality, we find that the condition is equivalent to

$$
\sqrt{\frac{1}{\pi \delta\left[2 \pi\left(k^{2}+1\right)-(k+1)\right]} M}<\sqrt{\frac{\alpha^{2}}{\pi \delta\left[2 \pi\left(k^{2}+1\right)+\alpha^{2}(k+1)\right]} M} .
$$

Hence, the inequality is satisfied for $M$ large enough if

$$
\frac{1}{2 \pi\left(k^{2}+1\right)-(k+1)}<\frac{\alpha^{2}}{2 \pi\left(k^{2}+1\right)+\alpha^{2}(k+1)} .
$$

Since

$$
\frac{1}{2 \pi\left(k^{2}+1\right)-(k+1)}<\frac{1}{2 \pi\left(k^{2}+1\right)-2 \pi(k+1)}
$$

and

$$
\frac{\alpha^{2}}{2 \pi\left(k^{2}+1\right)+2 \pi \alpha^{2}(k+1)}<\frac{\alpha^{2}}{2 \pi\left(k^{2}+1\right)+\alpha^{2}(k+1)}
$$

(A.6) is implied by

$$
\frac{1}{2 \pi\left(k^{2}+1\right)-2 \pi(k+1)}<\frac{\alpha^{2}}{2 \pi\left(k^{2}+1\right)+2 \pi \alpha^{2}(k+1)}
$$

or equivalently by

$$
\left(\alpha^{2}-1\right) k^{2}-2 \alpha^{2} k-\alpha^{2}-1>0 .
$$

Since $\alpha \in(1, \sqrt{2})$, it follows that a sufficient condition for (A.6) to hold is

$$
k>\frac{\alpha^{2}+\sqrt{2 \alpha^{4}-1}}{\alpha^{2}-1} .
$$


This concludes the proof that for any $\varepsilon \in(0,1)$, there exist $k, \delta, M$ such that the function

$$
R_{k, \delta, M}(t):=M+\delta \sin (2 \pi k t)+\delta \sin (2 \pi t)
$$

is in $\widetilde{\mathcal{R}}$. Moreover, for any

$$
\varepsilon \in\left(0, \sqrt{1-\frac{1}{(\pi-1)^{2}}}\right)
$$

we have $\alpha^{2} \in\left(\frac{\pi}{\pi-1}, 2\right)$, and the previous arguments can be repeated to show that there exist $\delta, M$ such that the function $R_{k, \delta, M}(t)$ is in $\widetilde{\mathcal{R}}$ for $k=1$ (it is enough to check (A.6) with $k=1$ ).

\section{B. Some Results of Aubry-Mather Theory}

In this section, we gather the results from Aubry-Mather theory that are used in the paper. For the proofs, we refer to $[3,4,11,27,28]$.

Consider the cylinder $\mathbb{A}=\mathbb{T} \times \mathbb{R}$ and a strip $\Sigma=\mathbb{T} \times(a, b)$ with $-\infty \leq$ $a<b \leq+\infty$. Let $S: \Sigma \rightarrow \mathbb{A}$, be a $C^{2}$-embedding and denote $S(x, y)=(\bar{x}, \bar{y})$ and $S^{n}(x, y)=\left(x_{n}, y_{n}\right)$.

In the following, we will tacitly consider the lift of $S$ to the universal cover $\mathbb{R}^{2}$ of $\mathbb{A}$ where $x \in \mathbb{R}, \bar{x}(x+1, y)=\bar{x}(x, y)+1$ and $\bar{y}(x+1, y)=\bar{y}(x, y)$. With some abuse, we will use the same notation for $S$ and its lift, and the correct interpretation should be clear from the context.

We suppose that $S$ is exact symplectic and twist. The exact symplectic condition requires the existence of a $C^{2}$ function $V: \Sigma \rightarrow \mathbb{R}$ such that

$$
\bar{y} d \bar{x}-y d x=d V(x, y) \text { in } \Sigma,
$$

and the (positive) twist condition reads

$$
\frac{\partial \bar{x}}{\partial y}>0 \quad \text { in } \Sigma .
$$

A negative twist condition would give analogous results. If $\Sigma=\mathbb{A}$, we also suppose that $S$ preserves the ends of the cylinder that is

$$
\bar{y} \rightarrow \pm \infty \quad \text { as } y \rightarrow \pm \infty \text { uniformly in } x,
$$

and twists each end infinitely that is

$$
\bar{x}-x \rightarrow \pm \infty \quad \text { as } y \rightarrow \pm \infty \text { uniformly in } x .
$$

Note that the exact symplectic condition implies that $S$ is orientation preserving and preserves the two-form $d y \wedge d x$.

For this class of maps, the following result is well known $[4,28]$. In the following, we denote the partial derivative of $h$ with respect to the $i$-th variable by $h_{i}$.

Proposition B.1. Given $\Omega:=\left\{(x, \bar{x}) \in \mathbb{R}^{2}: \bar{x}(x, a) \leq \bar{x} \leq \bar{x}(x, b)\right\}$, there exists a $C^{2}$ function $h: \Omega \rightarrow \mathbb{R}$ such that

(i) $h(x+1, \bar{x}+1)=h(x, \bar{x})$ in $\Omega$, 
(ii) $h_{12}(x, \bar{x})<0$ in $\Omega$,

(iii) for $(x, y) \in \Sigma$ we have $S(x, y)=(\bar{x}, \bar{y})$ if and only if

$$
\left\{\begin{array}{l}
h_{1}(x, \bar{x})=-y \\
h_{2}(x, \bar{x})=\bar{y}
\end{array}\right.
$$

Conversely, for $\Omega^{\prime}:=\left\{(x, \bar{x}) \in \mathbb{R}^{2}: a^{\prime} \leq \bar{x}-x \leq b^{\prime}\right\}$ let $h^{\prime}: \Omega^{\prime} \rightarrow \mathbb{R}$ be a $C^{2}$ function such that

(i) $h^{\prime}(x+1, \bar{x}+1)=h^{\prime}(x, \bar{x})$ in $\Omega^{\prime}$,

(ii) $h_{12}^{\prime}(x, \bar{x})<0$ in $\Omega^{\prime}$,

then, the equations

$$
\left\{\begin{array}{l}
h_{1}^{\prime}(x, \bar{x})=-y \\
h_{2}^{\prime}(x, \bar{x})=\bar{y}
\end{array}\right.
$$

define implicitly on $\Sigma^{\prime}:=\mathbb{T} \times\left(-h_{1}^{\prime}\left(x, \bar{x}+a^{\prime}\right),-h_{1}^{\prime}\left(x, \bar{x}+b^{\prime}\right)\right)$ a $C^{2}$ exact symplectic twist embedding $S^{\prime}: \Sigma^{\prime} \rightarrow \mathbb{A}$.

Remark B.2. If $\Sigma=\mathbb{A}$, the fact that $S$ preserves and twists each end infinitely implies that $\Omega=\mathbb{R}^{2}$. The condition $h_{12}(x, \bar{x})<0$ is related to the twist condition. Actually, the twist implies that we can write $y=y(x, \bar{x})$ and one gets that

$$
h_{12}(x, \bar{x})=-\left(\frac{\partial \bar{x}}{\partial y}(x, y(x, \bar{x}))\right)^{-1} .
$$

The function $h\left(\right.$ or $\left.h^{\prime}\right)$ is called generating function and gives an equivalent implicit definition of the diffeomorphism $S$. From this proposition, one has that a sequence $\left(x_{n}, y_{n}\right)_{n \in \mathbb{Z}}$ such that $\left(x_{n}, y_{n}\right) \in \Sigma$ for every $n \in \mathbb{Z}$ is an orbit of $S$ if and only if for every $n \in \mathbb{Z}$ one has $\left(x_{n}, x_{n+1}\right) \in \Omega$ and

$$
\begin{aligned}
& h_{2}\left(x_{n-1}, x_{n}\right)+h_{1}\left(x_{n}, x_{n+1}\right)=0, \\
& y_{n}=-h_{1}\left(x_{n}, x_{n+1}\right) .
\end{aligned}
$$

From now on, we consider the case $\Sigma=\mathbb{A}$. Actually, the following extension result (see, for example, $[22,28]$ ) guarantees that we can always extend an exact symplectic diffeomorphism defined on a strip to one defined on the cylinder.

Lemma B.3. Let $h$ be a $C^{2}$ generating function defined on $\Omega=\left\{(x, \bar{x}) \in \mathbb{R}^{2}\right.$ : $a \leq \bar{x}-x \leq b\}$ such that $h_{12} \leq \delta<0$ on $\Omega$. Then there exists a generating function $\tilde{h}$ defined on $\mathbb{R}^{2}$ such that $h=\tilde{h}$ on $\Omega$ and $\tilde{h} \leq \delta<0$ on $\mathbb{R}^{2}$. Moreover, $\tilde{h}=\frac{1}{2}(\bar{x}-x)^{2}$ on $\mathbb{R}^{2} \backslash \Omega_{\beta}$, being $\Omega_{\beta}=\left\{(x, \bar{x}) \in \mathbb{R}^{2}: a-\beta \leq \bar{x}-x \leq b+\beta\right\}$.

Let $\Sigma=\mathbb{A}$, we recall the variational characterisation of the orbits of $S$ in terms of the action

$$
H_{\ell k}\left(x_{\ell}, \ldots, x_{k}\right)=\sum_{n=\ell}^{k-1} h\left(x_{n}, x_{n+1}\right) .
$$


It is well known that solutions of (B.1) (and hence orbits of $S$ ) are in 1-1 correspondence with stationary points of $H_{\ell k}$ with respect to variations fixing the endpoints $x_{\ell}, x_{k}$. In the following, we are interested in minimal orbits, i.e. orbits $\left(x_{n}, y_{n}\right)_{n \in \mathbb{Z}}$ of $S$ such that for every pair of integers $h<k$ and for every sequence of real numbers $\left(x_{n}^{*}\right)_{\ell \leq n \leq k}$ such that $x_{\ell}^{*}=x_{h}$ and $x_{k}^{*}=x_{k}$, it holds

$$
H_{\ell k}\left(x_{\ell}, \ldots, x_{k}\right) \leq H_{\ell k}\left(x_{\ell}^{*}, \ldots, x_{k}^{*}\right) .
$$

Moreover, we recall that an orbit $\left(x_{n}, y_{n}\right)_{n \in \mathbb{Z}}$ of $S$ has rotation number $\omega \in \mathbb{R}$ if

$$
\lim _{n \rightarrow \infty} \frac{x_{n}}{n}=\omega
$$

It is well known that minimal orbits are monotone, that is only one of the following is satisfied:

$$
\begin{aligned}
& x_{n}<x_{n+1} \text { for every } n \in \mathbb{Z}, \\
& \quad x_{n}=x_{n+1} \text { for every } n \in \mathbb{Z}, \quad x_{n}>x_{n+1} \text { for every } n \in \mathbb{Z} .
\end{aligned}
$$

Moreover, if it has rotation number $\omega$, then it satisfies the following estimate for every $n, m \in \mathbb{Z}$ :

$$
\left|x_{n}-x_{m}-(n-m) \omega\right| \leq 1 .
$$

Finally we recall that an invariant set of $S$ is said to be minimal and with rotation number $\omega$ if it is made of minimal orbits with rotation number $\omega$ and that the term invariant curve of $S$ refers to a curve $\Gamma \subset \Sigma$ homotopic to $\{(x, y) \in \mathbb{A}: y=k$, for some $k \in \mathbb{R}\}$ and such that $S(\Gamma)=\Gamma$.

The following theorem gives the existence of minimal orbits with rotation number.

Theorem B.4 [ [4,27]]. Let $h: \mathbb{R}^{2} \rightarrow \mathbb{R}$ be a $C^{2}$ generating function such that

(i) $h(x+1, \bar{x}+1)=h(x, \bar{x})$ in $\mathbb{R}^{2}$,

(ii) $h_{12}(x, \bar{x}) \leq \delta<0$ in $\mathbb{R}^{2}$

and let $S$ be the corresponding diffeomorphism. For a fixed $\omega \in \mathbb{R}$

- if $\omega=p / q \in \mathbb{Q}$, then there exists a minimal orbit $\left(x_{n}, y_{n}\right)_{n \in \mathbb{Z}}$ of $S$ such that $\left(x_{n+q}, y_{n+q}\right)=\left(x_{n}+p, y_{n}\right)$

- if $\omega \in \mathbb{R} \backslash \mathbb{Q}$, then there exists a minimal invariant set $M_{\omega}$ of rotation number $\omega$ such that $M_{\omega}$ is the graph of a Lipschitz function $u: \pi\left(M_{\omega}\right) \rightarrow$ $\mathbb{R}$. Moreover, $M_{\omega}$ is either an invariant curve or a Cantor set.

The following corollary gives an equivalent interpretation of the result and has been proven in [26] (see also [23]).

Corollary B.5. For each $\omega \in \mathbb{R}$, there exist two functions $\phi, \eta: \mathbb{R} \rightarrow \mathbb{R}$ such that for every $\xi \in \mathbb{R}$

$$
\begin{gathered}
\phi(\xi+1)=\phi(\xi)+1, \quad \eta(\xi+1)=\eta(\xi), \\
S(\phi(\xi), \eta(\xi))=(\phi(\xi+\omega), \eta(\xi+\omega))
\end{gathered}
$$

where $\phi$ is monotone (strictly if $\omega \in \mathbb{R} \backslash \mathbb{Q}$ ) and $\eta$ is of bounded variation. Moreover, $\phi$ and $\eta$ have the same points of continuity and if $\xi$ is a point of continuity, then so are $\xi \pm \omega$ and $\xi \pm 1$. 
For irrational rotation numbers $\omega$, Theorem B.4 leaves open the possibility for the minimal set $M_{\omega}$ to be an invariant curve or not. To prove what is the case for a given $\omega$ is of fundamental importance to prove the existence of chaotic motion for the diffeomorphism $S$. We recall the following result by Forni.

Let us fix $\omega \in \mathbb{R} \backslash \mathbb{Q}$ and denote by $\sigma_{\omega}$ the unique $S$-invariant ergodic Borel probability measure supported on $M_{\omega}$.

Theorem B.6 [ [11]]. Let $S$ be a $C^{2}$ diffeomorphism of the cylinder $\mathbb{A}$ as in Theorem B.4. Suppose that $S$ does not admit any invariant curve of rotation number $\omega$. Then there exists an $S$-invariant ergodic Borel probability measure $\mu_{\omega}$ with positive metric entropy. Moreover, $\mu_{\omega}$ can be chosen arbitrarily close to $\sigma_{\omega}$ in the sense of the weak topology on the space of compactly supported Borel probability measures on $\mathbb{A}$.

Finally we recall a result to prove whether the set $M_{\omega}$ is an invariant curve or not.

Proposition B.7 [ [25]]. Let $S$ be a $C^{2}$ diffeomorphism of the cylinder $\mathbb{A}$ as in Theorem B.4, and let $\Gamma$ be an invariant curve of $S$. Then

(i) $\Gamma$ is a minimal set and each orbit on $\Gamma$ has the same rotation number;

(ii) for any orbit $\left(x_{n}, y_{n}\right)$ on $\Gamma$, it holds

$$
a\left(x_{n-1}, x_{n}, x_{n+1}\right):=h_{22}\left(x_{n-1}, x_{n}\right)+h_{11}\left(x_{n}, x_{n+1}\right)>0, \quad \forall n \in \mathbb{Z} .
$$

\section{References}

[1] Angenent, S.B.: Monotone recurrence relations, their Birkhoff orbits and their topological entropy. Ergodic Theory Dyn. Syst. 10, 15-41 (1990)

[2] Angenent, S.B.: A remark on the topological entropy and invariant circles of an area preserving twist map. In: McGehee, R., Meyer, K.R. (eds.) Twist Mappings and their Applications, pp. 1-5. Springer, New York (1992)

[3] Aubry, S., Le Daeron, P.Y.: The discrete Frenkel-Kontorova model and the devil's staircase. Phys. D 7, 240-258 (1983)

[4] Bangert, V.: Mather sets for twist maps and geodesics on tori. In: Dynamics Reported, Vol. 1 pp. 1-56. Wiley, Chichester (1988)

[5] Blocki, J., Boneh, Y., Nix, J.R., Randrup, J., Robel, M., Sierk, A.J., Swiatecki, W.J.: One-body dissipation and the super-viscidity of nuclei. Ann. Phys. 113, 330-86 (1978)

[6] Burgio, G.F., Baldo, M., Rapisarda, A.: Chaoticity in vibrating nuclear billiards. Phys. Rev. C 52, 2475 (1995)

[7] del-Castillo-Negrete, D., Greene J.M., Morrison P.J.: Area preserving nontwist maps: periodic orbits and transition to chaos. Phys. D Nonlinear Phenomena 91, 1-23 (1996)

[8] Delshams, A., de la Llave, R.: KAM theory and a partial justification of Greene's criterion for nontwist maps. SIAM J. Math. Anal. 31, 1235-1269 (2000) 
[9] Dettmann, C.P., Fain, V., Turaev, D.: Splitting of separatrices, scattering maps, and energy growth for a billiard inside a time-dependent symmetric domain close to an ellipse. Nonlinearity 31, 667-700 (2018)

[10] Fermi, E.: On the origin of the cosmic radiation. Phys. Rev. 15, 1169-1174 (1949)

[11] Forni, G.: Construction of invariant measures supported within the gaps of Aubry-Mather sets. Ergodic Theory Dyn. Syst. 16, 51-86 (1996)

[12] Gelfreich, V., Rom-Kedar, V., Turaev, D.: Fermi acceleration and adiabatic invariants for non-autonomous billiards. Chaos 22, 033116 (2012)

[13] Glanz, J.: Time-reversed sound waves resonate among physicists. Science 265, 474-75 (1994)

[14] Haro, Á.: Converse KAM theory for monotone positive symplectomorphisms. Nonlinearity 12, 1299-1322 (1999)

[15] Koiller, J., MDarkarian, R., Oliffson Kamphorst, S., Pinto de Carvalho S.: Timedependent billiards. Nonlinearity 8, 983-1003 (1995)

[16] Kunze, M., Ortega, R.: Complete orbits for twist maps on the plane: the case of small twist. Ergodic Theory Dyn. Syst. 31, 1471-1498 (2011)

[17] Laederich, S.: Invariant curves and time dependent potentials. Levi. M. Ergodic Theory Dyn. Syst. 11, 365-78 (1991)

[18] MacKay, R.S., Meiss, J.D., Stark, J.: Converse KAM theory for symplectic twist maps. Nonlinearity 2, 555-570 (1989)

[19] MacKay, R.S., Percival, I.C.: Converse KAM: theory and practice. Commun. Math. Phys. 98, 469-512 (1985)

[20] Marò, S.: Coexistence of bounded and unbounded motions in a bouncing ball model. Nonlinearity 26, 1439-1448 (2013)

[21] Marò, S.: A mechanical counterexample to KAM theory with low regularity. Phys. D 283, 10-14 (2014)

[22] Marò, S.: Chaotic dynamics in an impact problem. Ann. Henri Poincaré 16, 1633-1650 (2015)

[23] Marò, S.: Relativistic pendulum and invariant curves. Discrete Contin. Dyn. Syst. 35, 1139-1162 (2015)

[24] Marò, S.: Diffusion and chaos in a bouncing ball model. Z. Angew. Math. Phys. 71, 78 (2020)

[25] Mather, J.N.: Glancing billiards. Ergodic Theory Dyn. Syst. 2, 397-403 (1982)

[26] Mather, J.N.: Existence of quasi-periodic orbits for twist homeomorphisms of the annulus. Topology 21, 457-67 (1982)

[27] Mather, J.N.: Variational construction of orbits of twist diffeomorphisms. J. Am. Math. Soc. 4, 207-263 (1991)

[28] Mather, J.N., Forni, G.: Action minimizing orbits in Hamiltonian systems. In: Graffi S. (eds) Transition to Chaos in Classical and Quantum Mechanics. Lecture Notes in Mathematics, vol. 1589. Springer, Berlin (1994)

[29] Oliffson Kamphorst, S., Pinto de Carvalho, S.: Bounded gain of energy on the breathing circle billiard. Nonlinearity 12, 1363-1371 (1999)

[30] Ortega, R.: Asymmetric oscillators and twist mappings. J. London Math. Soc. 2(53), 325-342 (1996)

[31] Pais, A.: Niels Bohr's Times, in Physics, Philosophy and Polity. Oxford University Press (1991) 
[32] Siegel, C., Moser, J.: Lectures on Celestial Mechanics. Springer (1971)

[33] Zharnitsky, V.: Instability in Fermi-Ulam ping-pong problem. Nonlinearity 11, 1481 (1998)

Claudio Bonanno and Stefano Marò

Dipartimento di Matematica

Università di Pisa

Largo Bruno Pontecorvo 5

56127 Pisa

Italy

e-mail: claudio.bonanno@unipi.it;

stefano.maro@unipi.it

Communicated by Dmitry Dolgopyat.

Received: January 30, 2021.

Accepted: July 15, 2021. 